\begin{tabular}{ll}
\hline FORSCHUNG & \\
ZEISCHRIF FUR \\
BETRIEBSWRTSCHAFT \\
OGabler-Verlag 2008
\end{tabular}

\title{
Die Macht des Defaults - Wirkung von Empfehlungen und Vorgaben auf das individuelle Entscheidungsverhalten
}
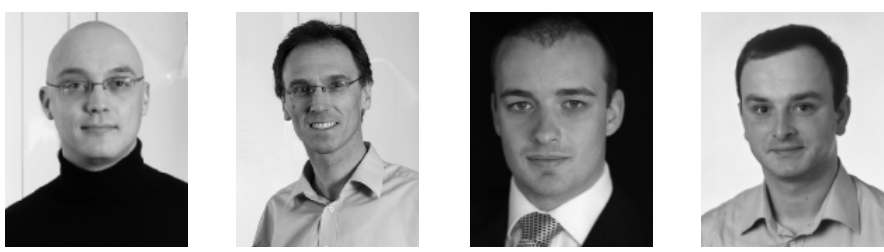

Benjamin Polak, Andreas Herrmann, Mark Heitmann, Martin Einhorn

\section{Überblick}

- Bei der Wahl zahlreicher Erzeugnisse, wie etwa Pkw oder Computer, ist der Kunde im Rahmen der Produktspezifikation mit Empfehlungen und Vorgaben der Hersteller und Händler (Defaults) konfrontiert. Beispielsweise ist bei der online-Konfiguration eines Fahrzeugs für alle zwingend erforderlichen Merkmale (z. B. Farbe) jeweils ein Default (d. h. eine Ausprägung, z. B. Silber) vorgegeben. Zudem geben die Anbieter Empfehlungen bezüglich bestimmter Merkmalsausprägungen ab, an denen sich die Nachfrager orientieren.

- Der grundsätzliche Einfluss von Defaults auf das Entscheidungsverhalten von Individuen ist in der Literatur unbestritten. Gleichwohl sind wichtige Fragen etwa nach dem Effekt unterschiedlicher Default-Varianten noch nicht beantwortet.

- Anhand einer empirischen Studie, in deren Mittelpunkt ein Car-Konfigurator steht, kann gezeigt werden, wie verschiedene Defaults das Entscheidungsverhalten der Individuen beeinflussen. Die Befunde erlauben Rückschlüsse über den Prozess der Herausbildung von Produktpräferenzen.

- Für die Hersteller und Händler ergeben sich konkrete Hinweise für die Gestaltung von Empfehlungen und Vorgaben. Der Wissenschaftler erhält einen Eindruck über die Relevanz von Defaults bei der Konfiguration von Erzeugnissen. Zudem lassen sich Erkenntnisse über den Entscheidungsprozess von Individuen, insbesondere im Hinblick auf die Wahrnehmung und Beurteilung von Produktmerkmalen und deren Ausprägungen ableiten.

Keywords Default $\cdot$ decision making $\cdot$ experimental design $\cdot$ car configurator

Eingegangen: 7. Januar 2007

JEL: M30, M31

Dipl.-Inf. Benjamin Polak ( $\square$ )

Universität St. Gallen, Forschungsstelle für Business Metrics und Audi Lab for Market Research,

Guisanstrasse 1a, CH-9000 St. Gallen. Email: benjamin.polak@unisg.ch, URL: http://www.zbm.unisg.ch.

Prof. Dr. Andreas Herrmann $(\square)$

Universität St. Gallen, Forschungsstelle für Business Metrics und Audi Lab for Market Research,

Guisanstrasse 1a, CH-9000 St. Gallen. Email: andreas.herrman@unisg.ch, URL: http://www.zbm.unisg.ch.

Prof. Dr. Mark Heitmann ( $\bowtie)$

Christian-Albrechts-Universität Kiel, Department für Marketing, Westring 425, 24118 Kiel.

Email: heitmann@bwl.uni-kiel.de, URL:www.bwl.uni-kiel.de.

Dr. Martin Einhorn $(\bowtie)$

Koordinator Trendforschung für die Audi AG bis Mai 2008. Leiter Marktforschung der Dr. Ing. h.c. F. Porsche AG ab Juni 2008. 


\section{A. Relevanz des Defaults für das Entscheidungsverhalten}

Es ist unbestritten, dass Hersteller- und Händlerempfehlungen bei der Auswahl von Produkten und Dienstleistungen eine zentrale Rolle spielen (vgl. Boyle et al., 1992; Brown, 1990). Viele Kunden orientieren sich beim Erwerb von Erzeugnissen aus vielerlei Gründen willentlich oder auch nicht gezielt an diesen Empfehlungen (vgl. Del Veccio et al., 2003; Bearden/Netemeyer/Teel, 1989; Fitzsimons/Lehmann, 2004). Insofern ist es nicht verwunderlich, dass Hersteller und Händler ihre Empfehlungen über Produkte und Services explizit oder implizit im persönlichen Gespräch mit dem Kunden oder in Werbebotschaften einfließen lassen. Eine Literaturtradition liegt vor, aus der sich konkrete Hinweise für die Art und Weise der Präsentation von Empfehlungen im Rahmen der Kommunikation mit den Kunden ergeben (vgl. den Überblick bei Gedenk, 2002, S. 278 ff.). Viele dieser Arbeiten sind abgestützt auf empirischen Untersuchungen, die die Wirkung der Ratschläge von Herstellern und Händlern auf das Entscheidungsverhalten der Individuen verdeutlichen.

Bei einigen Erzeugnissen kommen diese Empfehlungen jedoch nicht durch die Beteiligten (Händler oder Hersteller) selbst zum Ausdruck; vielmehr existieren neben diesen weichen Defaults (Hersteller- und Händlerempfehlungen) auch harte. Letztere verkörpern jene Optionen, die der Kunde automatisch erhält, sofern er sich nicht ausdrücklich für andere entscheidet (vgl. Brown/Krishna, 2004, S. 529). Ein harter Default lässt sich als Voreinstellung bei den Merkmalsausprägungen eines Produkts bzw. einer Dienstleistung kennzeichnen (vgl. Park/Jun/MacInnis, 2000, S. 191 ff.). Entspricht diese Voreinstellung nicht den Wünschen des Kunden, muss er diese willentlich durch eine andere Option ersetzen. Charakteristisch hierbei ist die Anstrengung des Individuums, den Default abwählen zu müssen, um ein anderes Produkt bzw. eine andere Merkmalsausprägung zur besseren Befriedigung seiner Bedürfnisse zu erhalten.

Ein Blick auf das Geschehen in vielen Märkten zeigt, dass Individuen bei einer Fülle von Produktwahlhandlungen mit weichen und harten Defaults konfrontiert sind. Man denke etwa an die Speisekarte eines Restaurants. Zumeist sind bestimmte Kombinationen aus z. B. Fleisch oder Fisch, Gemüse und Beilage empfohlen (oder vorgegeben). Dabei hängt es von der Flexibilität des Kochs ab, ob diese Empfehlungen (weiche Defaults) auch Vorgaben (harte Defaults) sind. In jedem Fall muss der Gast den Kellner ansprechen, ihn möglicherweise überzeugen, will er vom Menuvorschlag abweichen und z. B. Reis anstelle von Kartoffeln bestellen. Ein anderes Beispiel ist ein Reiseveranstalter, der Städtereisen offeriert, die einen Flug, eine Unterkunft und ein Besuchsprogramm umfassen. Dabei besteht der Default für die Unterkunft aus einem Standarddoppelzimmer (harter Default). Wünscht der Kunde z. B. ein Luxusdoppelzimmer, muss er auf dem Anmeldeformular die vorgegebene Zimmerkategorie durch die bevorzugte ersetzen. Auch ist ein Hinweis auf ein Konzert denkbar, das (so die Argumentation des Reiseveranstalters) dem Gast einen Eindruck über die kulturellen Besonderheiten der besuchten Stadt vermittelt (weicher Default). Ein Versicherungsunternehmen offeriert für Reisende ins Ausland ein Versicherungspaket, das alle Standardleistungen eines ausländischen Krankenhauses beinhaltet. Möchte das Individuum darüber hinaus einen Rücktransport ins Heimatland, muss es sich gegen das Basisprodukt entscheiden und ein erweitertes Paket wählen (harter Default). Zudem könnte z. B. ein Prominenter (auf einem Prospekt) darauf hinweisen, 
dass die Chefarztbehandlung in vielen Ländern für die schnelle und vollständige Genesung bedeutsam ist (weicher Default).

Diese Beispiele zeigen, dass Individuen in zahlreichen Lebenssituationen auf weiche und harte (häufig auch kombinierte) Defaults treffen und ihre Entscheidungen über Erzeugnisse unter expliziter oder (zumeist wohl) impliziter Berücksichtigung dieser Empfehlungen und Vorgaben fällen. In ersten Arbeiten hierzu konnte nachgewiesen werden, dass Defaults unabhängig von der Gattung des betrachteten Produkts bzw. Produktmerkmals und unabhängig von den Persönlichkeitsmerkmalen der betroffenen Personen auf das Entscheidungsverhalten wirken (vgl. Bellman/Johnson/Lohse, 2001; Johnson/Bellman/Lohse, 2002; Madrian/Shea, 2001; Schkade/Kahneman, 1998). Brown und Krishna (2004, S. 533 ff.) knüpften an diese verhaltenswissenschaftlichen Arbeiten an und analysierten das Verhalten von Kunden, die die Absicht eines Unternehmens durchschauen, durch den Einsatz von Defaults Absatz und Umsatz zu verbessern. Park, Jun und MacInnis (2000, S. 191 ff.) interessierten sich für die Unterschiede im tatsächlich erworbenen Produkt, die sich aus einer Produktspezifikation ausgehend von einem ausgestatteten Modell (d. h. nicht gewünschte Produktmerkmale müssen abgewählt werden) im Vergleich zu einer Gestaltung auf Basis eines „nackten“ Modells (d. h. gewünschte Produktmerkmale müssen gewählt werden) ergeben. Obgleich der Effekt von Defaults auf das Entscheidungsverhalten von Individuen unbestritten ist, sind zahlreiche Fragen nach wie vor unbeantwortet:

(1) In vielen Fällen (z. B. bei Pkw) sind harte Defaults bei jener Ausprägung eines Produktmerkmals gesetzt, die eine geringe Qualität und einen niedrigen Preis aufweist (vgl. dazu die Car-Konfiguratoren der Automobilunternehmen). Folglich liegt es nahe, die Wirkung von Defaults zu analysieren, die stufenweise bei Ausprägungen mit besserer Qualität und höherem Preis angesiedelt sind.

(2) Daneben besitzt auch die Quelle, aus der der Default stammt, eine zentrale Bedeutung zur Erklärung des Entscheidungsverhaltens der Individuen. Es ist nahe liegend, dass verschiedene Absender (z. B. Hersteller, Händler, andere Kunden) von Empfehlungen bei den Adressaten unterschiedliche Wirkungen auf die Wahl eines Erzeugnisses oder eines Produktmerkmals bzw. dessen Ausprägung entfalten.

(3) Ohne Zweifel wählt nicht jedes Individuum den vom Hersteller bzw. Händler gesetzten Default. Gleichwohl ist denkbar, dass ein Default als Ankerpunkt wirkt und Kunden veranlasst, sich für Produkte bzw. Merkmalsausprägungen ,,in seiner Nähe“ zu entscheiden.

(4) Zudem ist von Interesse, ob Defaults in Abhängigkeit von der Produktbeschaffenheit unterschiedlich wirken. Anknüpfend an die Arbeiten von Dhar und Wertenbroch (2000, S. 65 ff.) ist zu vermuten, dass von Defaults bei utilitaristischen bzw. hedonistischen Erzeugnissen ein unterschiedlicher Effekt auf die Wahl eines Produkts bzw. eines Produktmerkmals ausgeht.

Zur Bewältigung dieser Aufgaben bietet sich folgende Vorgehensweise an: Zunächst richtet sich das Augenmerk auf das Phänomen Default und seine Erscheinungsformen. Daraufhin sollen Theorien zur Erklärung der Wirkung von Defaults aufgeworfen werden. Aus ihnen lassen sich im Folgenden Hypothesen über die Wirkung von Defaults auf das 
Entscheidungsverhalten der Individuen ableiten. Im Rahmen einer empirischen Untersuchung sollen die Hypothesen getestet werden. Aus den erzielten Ergebnissen ergeben sich Implikationen für die Produktgestaltung und Erkenntnisse über die Muster der individuellen Wahrnehmung und Beurteilung von Merkmalen und deren Ausprägungen.

\section{B. Zum Begriff Default und zu seinen Erscheinungsformen}

In Sinne einer einheitlichen Sprachregelung soll anknüpfend an die Literatur zwischen harten und weichen Defaults unterschieden werden (vgl. Hsee/Rottenstreich, 2004; Madrian/Shea, 2001).

\section{- Harter Default}

Im Einklang mit Brown und Krishna (2004, S. 530) lässt sich ein harter Default als Option auffassen, die das Individuum erhält, sofern es sich nicht willentlich für eine andere entscheidet. Ein Blick in viele Märkte zeigt, dass Hersteller und Händler immer wieder bei einzelnen Eigenschaftsausprägungen ihrer Erzeugnisse harte Defaults setzen. Ein Beispiel bilden Produktkonfiguratoren im Internet oder Produktspezifikationen via Formular (im Sinne eines offline-Konfigurators). In beiden Fällen muss der Kunde sukzessive über einzelne Merkmalsausprägungen entscheiden, um auf diesem Wege sein gewünschtes Erzeugnis zu gestalten. Zumeist greifen Hersteller und Händler (allein schon aus produktionstechnischen Erwägungen) in diesen Entscheidungsprozess ein, indem sie Defaults definieren, weil für die Erzeugung eines Produkts bei den meisten Merkmalen zwingend jeweils eine Ausprägung zu wählen ist. Dies ist beispielsweise dann der Fall, wenn ein Automobilunternehmen bestimmte Felgen zum Default erhebt, da dieses Merkmal für die Fahrzeugkonfiguration unerlässlich ist. Ergänzend hierzu dienen Defaults auch dazu, das Entscheidungsverhalten der Individuen in dem Sinne zu beeinflussen, die Meinungsbildung von Nachfragern im Entscheidungsprozess zu steuern. Hat ein Individuum vor der Produktwahl noch keine Präferenz für eine bestimmte Merkmalsausprägung entwickelt, kommt dem Default im Rahmen des Entscheidungsprozesses auch die Rolle eines Bezugspunkts zur Präferenzbildung zu. Johnson, Bellman und Lohse (2002) sowie Park, Jun und MacInnis (2000) legen empirische Untersuchungen vor, aus denen die Relevanz von harten Defaults zur Veränderung der Wahlwahrscheinlichkeit von Merkmalen und zur Herausbildung von Präferenzen für Merkmalsausprägungen bzw. Produkte hervorgeht.

Beispielsweise nutzt der Computerhersteller Dell diese Erkenntnis für die Gestaltung des Computer-Konfigurators. Gelangt ein Kunde bei der Spezifikation eines Dell-Computers zur Garantiezeit, ist diese mit drei Jahren vorgegeben. Wünscht der Kunde eine andere, muss er diese Vorgabe abwählen und seine bevorzugte Garantiezeit anklicken. Allerdings lassen sich viele Kunden von derartigen Vorgabe leiten, und sie scheuen die Mühe, vom Default abzuweichen (vgl. McKenzie/Liersch/Finkelstein, 2006). Zudem attestieren sie dem Hersteller oder Händler eine besondere Produktkompetenz, weshalb ein Abweichen vom Default als Risiko im Hinblick auf die Produktverwendung gilt (vgl. McKenzie, 2004). Offenbar eröffnet dieses Spiel mit dem Default den Anbietern neue 
Möglichkeiten, die Zahlungsbereitschaft ihrer Nachfrager besser abzuschöpfen und den Gewinn zu steigern.

Ein weiteres Beispiel für die verhaltensprägende Wirkung eines harten Defaults liefert ein Blick auf das Organspendeverhalten von Individuen (vgl. Johnson/Goldstein, 2003). Erstaunlicherweise variiert die Anzahl organspendewilliger Bürger an der Gesamtbevölkerung über verschiedene europäische Länder beträchtlich. In Österreich, den Niederlanden etc. erklären sich über 98 Prozent der Bürger im Todesfall zur Organspende bereit. Dagegen sind in Deutschland, der Schweiz etc. lediglich 2 Prozent der Bevölkerung willens, gegebenenfalls Organe zu spenden. Auf den ersten Blick mag man kulturelle Unterschiede als Erklärung für diesen überraschenden Befund anführen. Tatsächlich ist der Default bezüglich der Entscheidung für oder gegen die Organspende in den Ländern unterschiedlich gesetzt. In Österreich, den Niederlanden etc. ist jeder Bürger per Gesetz Organspender; er hat jedoch das Recht, eine Organspende abzulehnen und dies im Pass vermerken zu lassen. Umgekehrt sind die Bürger in Deutschland, der Schweiz etc. dem Gesetz entsprechend keine Organspender; es besteht jedoch die Möglichkeit, die Bereitschaft zur Organspende im Pass einzutragen. Beide gesetzlichen Rahmenbedingungen eröffnen den Individuen eine freie Entscheidung für oder gegen eine Organspende. Lediglich der Default ist in den beiden Entscheidungssituationen unterschiedlich gesetzt (vgl. Bellman/Johnson/Lohse, 2001).

\section{- Weicher Default}

Im Unterschied zum harten Default, der eine Vorentscheidung für eine Merkmalsausprägung durch den Anbieter verkörpert, repräsentiert der weiche lediglich eine Empfehlung. Dieser Ratschlag stellt keine Vorselektion dar, sondern dient allenfalls dazu, die Nachfrager auf eine bestimmte Merkmalsausprägung aufmerksam zu machen. Gleichwohl gilt auch in diesem Fall, dass sich viele Kunden von der Empfehlung leiten lassen, um etwa die mit einer Abweichung von ihr verbundene emotionale und kognitive Anstrengung zu vermeiden (vgl. McFarland/Challagalla/Shervani, 2006, S. 107 ff.; Ahearne/Gruen/Jarvis, 1999, S. 275 ff.). Darüber hinaus attestiert man dem Hersteller oder Händler Produktkompetenz, und auch das bereits angeführte Argument des empfundenen Risikos der Produktverwendung spielt eine Rolle.

Weiche Defaults treten in verschiedenen Erscheinungsformen auf; hierzu gehören etwa alle Hinweise des Anbieters auf die besondere Leistungsfähigkeit einzelner Merkmalsausprägungen (vgl. Bearden/Netemeyer/Teel, 1989, S. 476 ff.). Beispielsweise empfiehlt der Computerhersteller Dell seinen Kunden bei der Konfiguration eines PC, anstelle des integrierten Grafikchips eine dedizierte Grafikkarte zu wählen, um die vielfältigen Funktionen von Windows Vista besser nutzen zu können. Solche Hinweise lassen sich über Broschüren, klassische Print- und TV-Werbung oder durch eine Darbietung im Produktkonfigurator vermitteln. Häufig kommen Testimonials ins Spiel, um die Vertrauenswürdigkeit und Ausstrahlung einer Empfehlung zu unterstreichen. Im Pkw-Markt sind dies beispielsweise Rennfahrer, denen man die notwendige Kompetenz zuschreibt, Produktmerkmale und deren Ausprägungen vor dem Hintergrund bestimmter Verwendungszwecke einschätzen zu können. 
Eine andere Spielart weicher Defaults sind Empfehlungen, die etwa der Händler im Beratungsgespräch abgibt. Dieser verweist beispielsweise auf persönliche Erfahrungen (oder berichtet über die Erfahrungen Dritter) mit bestimmten Merkmalsausprägungen und setzt damit willentlich oder unwillentlich Defaults (vgl. Fitzsimons/Lehmann, 2004, S. 84 ff.). Aufgrund der ihm attestierten Fähigkeit, vielfältige Erfahrungen des Herstellers, anderer Kunden oder sachverständiger Dritter bezüglich einzelner Merkmalsausprägungen bündeln zu können, entfalten die (zumeist beiläufig im Gespräch) gesetzten Defaults eine beachtliche Wirkung auf das Entscheidungsverhalten. Aus empirischen Untersuchungen, in denen Händlerempfehlungen als Defaults interpretiert wurden, lässt sich die Erkenntnis ableiten, dass die Vermittlung von Defaults im Rahmen eines persönlichen (Beratungs-)Gesprächs ein effizientes Instrument zur Verhaltenssteuerung bildet (vgl. Ahearne/Gruen/Jarvis, 1999, S. 278 ff.; McFarland/Challagalla/Shervani, 2006, S. 112 ff.).

\section{Wirkung von Defaults auf das Entscheidungsverhalten}

Hält man sich die relevante Literatur vor Augen, fallen zwei wissenschaftliche Traditionen auf, aus denen sich Einsichten über die Wirkung von Defaults (gleichgültig ob hart oder weich) auf das Entscheidungsverhalten von Individuen ergeben. Ein erster Ansatz zielt darauf ab, die Entscheidung eines Individuums für eine als Default definierte Merkmalsausprägung zu erklären (vgl. Johnson et al., 1993; Johnson/Bellman/Lohse, 2002; Mardian/ Shea, 2001; McKenzie/Liersch/Finkelstein, 2006). Dabei interessiert allein die Wahl bzw. Nicht-Wahl des Defaults, ohne seine Wirkung auf die Wahrnehmung und Beurteilung aller anderen Merkmalsausprägungen zu betrachten (direkte Wirkung von Defaults). Ein davon abweichender Ansatz liefert Erkenntnisse über die Bedeutung des Defaults als Ankerpunkt im Entscheidungsprozess (vgl. Kahneman/Knetsch/Thaler, 1990; Kahneman/Tversky, 1979 und 1991; McKenzie/Nelson, 2003). Hier fungiert die zum Default erhobene Merkmalsausprägung als Gravitationspunkt, der Kunden veranlasst, eine Merkmalsausprägung ,rund um den Default“ zu wählen (indirekte Wirkung von Defaults). Es ist vorstellbar, dass selbst Individuen, die den Default nicht wählen, durch seine Existenz im Entscheidungsverhalten beeinflusst werden und sich für eine Merkmalsausprägung ,in der Nähe“ des Defaults entscheiden.

\section{Direkte Wirkung von Defaults auf das Entscheidungsverhalten}

Johnson und Goldstein (2003) nennen drei Gründe für die Wahl eines Defaults (vgl. zudem McKenzie/Nelson, 2003, S. 598 ff.; McKenzie, 2004, S. 879 ff.; Schkade/Kahneman, 1998, S. 342 ff.).

\section{- Wahl des Defaults aufgrund von „endorsement“"}

Zunächst lässt sich ein Default (ob hart oder weich ist unbedeutend) als Aufforderung zur Auswahl (,endorsement") auffassen. Kunden interpretieren einen wie auch immer präsentierten Default als Ratschlag des Herstellers oder Händlers, genau diese Merkmals- 
ausprägung zu wählen. Im Rahmen einer empirischen Untersuchung, die aus mehreren Experimenten besteht, bestätigen McKenzie, Liersch und Finkelstein (2006, S. 415 ff.) diese Facette von Defaults. Die Autoren zeigen, dass Individuen einen Default in der Tat als Anregung zur Auswahl einstufen, jedoch stets Vermutungen über die Gründe anstellen, die ein Unternehmen veranlassen, eine bestimmte Merkmalsausprägung zum Default zu erheben. Eine von Kunden immer wieder vorgebrachte Argumentation betrifft die Absicht eines Herstellers oder Händlers, eine ausgewählte Merkmalsausprägung als Wahlalternative ins Spiel zu bringen. Neben der Förderung von Absatz und Umsatz einzelner Merkmalsausprägungen zielen viele Unternehmen auch darauf ab, so die Vermutung vieler Kunden, dass ihre Erzeugnisse in einer bestimmten Beschaffenheit am Markt erscheinen. Insofern kommen Defaults in Betracht, um Erzeugnisse mit bestimmten Merkmalsausprägungen auszustatten und damit zur intendierten Produktpositionierung beizutragen.

Inwieweit der Default Akzeptanz bei den Kunden findet, hängt von der ,marketplace metacognition“ der Individuen ab (vgl. Wright, 2002; Campbell/Kirmani, 2000). Wie bereits angedeutet, stellen Kunden im Rahmen dieser „marketplace metacognition“ Überlegungen an, ob und inwieweit der Default in ihrem oder im Interesse des Unternehmens gesetzt ist (vgl. Friestad/Wright, 1995 und 1999). Einen Pol dieses Spektrums möglicher Einschätzungen bildet das Urteil, der Default sei allein deshalb definiert worden, um den Umsatz bzw. Gewinn des Unternehmens zu optimieren. In diesem Fall erzeugt der Default Reaktanz, da die Nachfrager davon ausgehen, der Anbieter wolle ihr Entscheidungsverhalten in seinem Sinne beeinflussen. Der andere Pol bringt die Einschätzung der Kunden zum Ausdruck, der Default trage dazu bei, den Entscheidungsprozess zu erleichtern und zu beschleunigen. Zudem könne der Einzelne zu einem Produkt gelangen, das seine Bedürfnisse umfassend befriedigt. Hier dominiert die Vorstellung, der Anbieter hätte die Verwendungszwecke der Nachfrager vor Augen und würde die Defaults allein nach diesem Kriterium bestimmen. Wo sich das Unternehmen aus Sicht der Kunden auf diesem Kontinuum befindet, hängt von dessen Vertrauenswürdigkeit ab (vgl. Jain/Posavac, 2001, S. 173 ff.).

\section{- Wahl des Defaults aufgrund von ,laziness“}

Der zweite Grund für die Wahl eines Defaults liegt in einer Reduktion der mit der Entscheidung verbundenen emotionalen und kognitiven Anstrengungen (,laziness“, vgl. hierzu auch Payne/Bettman/Johnson, 1993). Neueste Studien zeigen, dass Individuen Emotionen zur Produktbeurteilung und zur Entscheidungsfindung nutzen (vgl. dazu Levav et al., 2006 und 2007). Jedoch ist diese Ressource (Emotionen) begrenzt, so dass sie im Verlauf des Entscheidungsprozesses abnimmt und sich erst nach einer Erholungspause regeneriert. Je mehr Entscheidungen nacheinander zu treffen sind, desto weniger Emotionen verbleiben, um die letzten Entscheidungen zu treffen. Die abnehmende Menge dieser Ressource erlebt das Individuum als zunehmende Mühe, Entscheidungen etwa über die Produktspezifikation zu fällen. Diese als ,depletion effect“ bezeichnete Erscheinung lässt sich jedoch abmildern, sofern das Individuum bei einem bestimmten Merkmal keinen aufwendigen Auswahlprozess durchläuft, sondern sich gleich für den Default entscheidet (vgl. Desmeules, 2002, S. 10 ff.). Insofern erleichtert die Wahl von Defaults den 
Entscheidungsprozess, da der Kunde weniger von der Affektressource verbraucht, was er als schnelle, einfache Entscheidung, die ohne Mühe herbei geführt werden kann, erlebt.

\section{- Wahl des Defaults aufgrund von „loss aversion“}

Der dritte Grund für die Wahl von Defaults ergibt sich aus dem von Kahneman und Tversky $(1979,1991)$ beschriebenen Phänomen der Verlustaversion (,loss aversion“) (vgl. Novemsky/Kahneman, 2005a und 2005b). Der ,prospect“-Theorie zufolge beurteilen Individuen vorliegende Alternativen (auch Ausprägungen eines Merkmals) bezüglich eines Referenzpunkts (vgl. Schweitzer, 1994, S. 460 ff.; Samuelson/Zeckhauser, 1988, S. 14 ff.). Dabei gewichten sie negative Abweichungen (Verluste) von diesem Ankerpunkt stärker als positive (Gewinne). Diese Verlustaversion kommt in einer negativen Differenz zwischen der maximalen Zahlungsbereitschaft (willingness to pay, kurz WTP) und der minimal erwarteten Kompensation (willingness to accept, kurz WTA) für ein und dieselbe Option zum Ausdruck (d. h. WTP $<$ WTA). Beispielsweise sei ein Individuum betrachtet, das in einem Car-Konfigurator zwischen zwei Radios (einem Standardradio und einem neuartigen Stereogerät) wählen muss. Es liegt nahe, dass das zum Default erhobene Gerät ein Gefühl des Besitzes suggeriert, da dieses Radio vorselektiert ist und bereits in der Kalkulation des Fahrzeugpreises erscheint. Angenommen der Aufpreis des Stereogeräts (kurz P) liegt genau zwischen der WTP und der WTA für dieses Erzeugnis (d. h. WTP $<$ P $<$ WTA). In diesem Fall sollte die Wahl des Radiogeräts durch den Default determiniert sein. Liegt der Default beim Standardradio, wird das Individuum nicht das andere Gerät wählen, da der Aufpreis die Zahlungsbereitschaft überschreitet. Ist der Default hingegen beim Stereogerät gesetzt, wird sich der Kunde gegen das Standardradio entscheiden, weil der Preisnachlass die minimal erwartete Kompensation unterschreitet.

Obgleich die Wirkung von Defaults auf das Entscheidungsverhalten aus theoretischer Perspektive unbestritten ist (vgl. auch die Einschätzung von McKenzie/Liersch/Finkelstein, 2006), fehlt in der Praxis bislang eine systematische Auseinandersetzung mit diesem Thema. Polak, Herrmann und Heitmann (2006) zufolge sind die Defaults erstaunlicherweise bei vielen Produktmerkmalen bei den geringwertigsten Ausprägungen (z. B. $75 \mathrm{~kW}$ Motor beim Audi A4) gesetzt (harte Defaults). Dies gilt insbesondere für die zahlreichen Produktkonfiguratoren (z. B. für Uhren, Sportschuhe, Kosmetika, Hemden), mit deren Hilfe die Kunden online ihre gewünschten Erzeugnisse gestalten. Da in einigen Märkten zwischen der Konfiguration und der Bestellung derzeit noch ein Medienbruch besteht (d. h. es muss offline bestellt werden), sind die Händler in der Lage, im Beratungsgespräch eine von der geringwertigsten Ausprägung abweichende Empfehlung ins Spiel zu bringen (weiche Defaults). Unabhängig von der Art des Defaults ist in Anbetracht der vorliegenden Erkenntnisse zu vermuten, dass eine Verschiebung des Defaults von der geringwertigsten Ausprägung ( $75 \mathrm{~kW}$ ) zu einer höherwertigen (z. B. $100 \mathrm{~kW}$ ) den Umsatz entsprechend verschiebt. Da höherwertige Merkmalsausprägungen auch einen höheren Preis aufweisen, ist durch diese Verschiebung eine Steigerung des Umsatzes zu erwarten. Insofern gilt folgende Hypothese:

H1: Eine Verschiebung des Defaults von der geringwertigsten zu höherwertigen Merkmalsausprägungen führt zu einer Steigerung des Umsatzes. 
Wie erinnerlich bildet der harte Default eine Option, die das Individuum erhält, sofern es sich nicht willentlich für eine andere entscheidet. Diese Spielart ist durch einen Automatismus im Entscheidungsprozess gekennzeichnet mit der Folge, dass der Kunde lediglich die Vorgabe des Herstellers oder Händlers bestätigen muss. Dies äußert sich beispielsweise durch einen Klick auf die O.K.-Taste im online-Konfigurator oder durch eine Unterschrift auf einem Formular (z. B. für einen Kredit-, Spar- oder Versicherungsvertrag) in der offline-Welt. Obgleich auch bei weichen Defaults eine Merkmalsausprägung hervorgehoben ist, bedarf es trotzdem einer Entscheidung des Betroffenen für diese Option. Hierbei ist eine mentale Auseinandersetzung zumindest mit der zum Default erhobenen Merkmalsausprägung unerlässlich, da diese vom Kunden ausgewählt werden muss. In einer online-Konfiguration ist das hervorgehobene Merkmal anzuklicken und seine Wahl zu bestätigen. Bei einem Formular muss der Kunde an der entsprechenden Stelle ein Kreuz machen und diese Wahl zumindest am Ende der Spezifikation durch eine Unterschrift bestätigen.

Unter Rückgriff auf die drei Gründe zur Wahl eines Defaults, lässt sich ein unterschiedlicher Effekt von harten und weichen Defaults auf das Entscheidungsverhalten begründen. Bezüglich des ,endorsement“ liegt der Gedanke nahe, dass eine Vorgabe (harter Default) eine stärkere Verbindlichkeit signalisiert als eine Empfehlung (weicher Default). Der Hersteller oder Händler bringt durch die Vorgabe (zumindest implizit) seine Präferenz zum Ausdruck, die sich bereits in einer Vorselektion niedergeschlagen hat. Zudem befriedigen harte Defaults (im Vergleich zu weichen) das Bedürfnis nach „laziness“ besser. Bei ersteren ist lediglich eine Bestätigung erforderlich, wohingegen letztere eine explizite Selektion der zum Default erhobenen Merkmalsausprägung verlangen. Darüber hinaus ist die Empfindung, das Merkmal bereits zu besitzen (und es bei einer Entscheidung für ein anderes wieder aufgeben zu müssen), bei einem harten Default stärker als bei einem weichen. Insofern ist zu vermuten, dass das Gefühl der „loss aversion“ bei harten (im Vergleich zu weichen) Defaults besonders salient ist. In Anbetracht dieser Überlegungen liegt die folgende Hypothese auf der Hand:

H2a: Ein harter Default beeinflusst Individuen stärker als ein weicher Default bezüglich der Wahl der zum Default erhobenen Merkmalsausprägung.

Das Konzept der „marketplace metacognition“ liefert auch Hinweise darauf, dass Individuen die Empfehlungen anderer (zum Beispiel für bestimmte Erzeugnisse) im Hinblick auf die Absicht zur Beeinflussung überprüfen (vgl. Wright, 2002, S. 679 ff.). Friestad und Wright (1999, S. 185) gelangen auf Basis verhaltenswissenschaftlicher Studien zu der Erkenntnis, ,... that people draw on their beliefs about persuasion to cope with other people`s attempts to influence them ....". Die Fähigkeit, einen Versuch der Beeinflussung erkennen und abwehren zu können, bildet den Ausführungen zahlreicher Autoren zufolge eine zentrale sozio-kognitive Ressource (vgl. Campbell/Kirmani, 2000; Friestad/Wright, 1994 und 1995). Sie bewahrt das Individuum in Entscheidungssituationen davor, nicht unreflektiert einer Empfehlung zu folgen, sondern stets mögliche Interessen des Ratgebers zu berücksichtigen.

In der Literatur herrscht Einigkeit darüber, dass die Vertrauenswürdigkeit des Ratgebers ("source credibility") einen entscheidenden Einfluss auf die Bereitschaft des Ratsuchenden ausübt, sich an der Empfehlung zu orientieren (vgl. Jain/Posavac, 2001, S. 170 
ff.). Die Einschätzung der Vertrauenswürdigkeit mündet in einem Urteil darüber, ob und inwieweit der Ratschlag im Interesse des Ratsuchenden abgegeben wurde. Ist die "source credibility" nicht gegeben, besteht sowohl Misstrauen bezüglich der Intention der Empfehlung als auch im Hinblick auf ihre Güte und ihre Nützlichkeit (vgl. McGuire, 2000, S. 111 ff.). Gilt die Quelle des Ratschlags hingegen als glaubwürdig, geht der Adressat davon aus, dass die Empfehlung unter Berücksichtigung seiner Interessen formuliert wurde (vgl. Pornpitakpan, 2004). Daher lässt sich folgende Hypothese formulieren:

H2b: Die Wirkung eines weichen Defaults bezüglich der Wahl der zum Default erhobenen Merkmalsausprägung ist um so höher, je größer die Vertrauenswürdigkeit des Absenders der Empfehlung ist.

Im Rahmen einer Bestandsanalyse zeigen Polak, Herrmann und Heitmann (2006) die vielfältigen Spielarten von Defaults, die Unternehmen im Markt präsentieren. Insbesondere bezüglich der Default-Stufe (unterschiedliche Preis- bzw. Qualitätsniveaus als Defaults) und der Default-Quelle (Vorgabe und Empfehlungen verschiedener Quellen) finden sich mannigfaltige Erscheinungsformen. Dieser Befund steht im Einklang mit dem Erfahrungswissen von Managern, die zum kombinierten Einsatz von Default-Stufe und Default-Quelle raten. Dabei soll die Wirkung einer bestimmten Default-Stufe auf das Entscheidungsverhalten der Kunden durch ausgewählte Default-Quellen unterstützt werden. Allerdings liegt bislang keine theoretische Argumentation vor, aus der sich eine Hypothese über die zwischen der Default-Stufe und der Default-Quelle bestehende Interaktion ableiten lässt. Insofern soll an dieser Stelle im Sinne einer Exploration die Existenz einer Wechselwirkung zwischen diesen beiden Variablen überprüft werden, ohne dass eine theoretisch begründete Vorstellung über ihr Ausmaß und ihre Richtung besteht (vgl. Boyle et al., 1992, und Del Veccio et al., 2003).

Hält man sich die Schriften von Khan, Dhar und Wertenbroch (2004), Dubois, Laurent und Czellar (2004) sowie Kivetz und Simonson (2002a und 2002b) vor Augen, ist zu vermuten, dass Defaults in Abhängigkeit der Produktbeschaffenheit unterschiedlich wirken. Hierbei kommt insbesondere der Unterscheidung zwischen hedonistischen und utilitaristischen Produkten bzw. Produktmerkmalen eine zentrale Bedeutung zu. Erstere „,.. are multisensory and provide for experimental consumption, fun, pleasure, and excitement ...", während letztere „... are primarily instrumental and their purchase is motivated by functional product aspects ..." (Khan/Dhar/Wertenbroch, 2004, S. 4). Beispiele für hedonistische Produktmerkmale bei einem Automobil sind der CD-Player oder das Sound-System, wohingegen der Airbag oder die Air-Suspension zu den utilitaristischen gehören. Ohne Zweifel hängt es von der Ge- bzw. Verbrauchssituation ab, ob und inwieweit ein Produkt bzw. ein Produktmerkmal als hedonistisch oder utilitaristisch gilt (vgl. Pham, 1998, S. 147 ff.). Beispielsweise verkörpert ein Handy ein utilitaristisches Gut, sofern es dazu dient, in einer Notsituation Hilfe anzufordern. Kommt es hingegen als "elektronisches Accessoire" in Betracht, das im Hinblick auf Farbe und Form modischen Ansprüchen genügen muss, repräsentiert das Handy ein hedonistisches Erzeugnis.

Aus entscheidungstheoretischen Arbeiten ist bekannt, dass eine Person ein Produktmerkmal als umso wichtiger einstuft, je leichter sie es wahrnehmen kann (vgl. Shiv/ Huber, 2000, S. 208 ff.). Da hedonistische Merkmale in der Regel leichter "ins Auge springen" als utilitaristische, sollten sie bei der Produktwahl eine zentrale Rolle spielen 
(vgl. Dhar/Wertenbroch, 2000, S. 62). Wie Roese (1997, S. 137 ff.) und Sanna (1999, S. $342 \mathrm{ff}$.) zeigen, ist die vom Individuum erlebte Verlustaversion bei wichtigen Merkmalen stärker als bei unwichtigen. Den empirischen Beleg für diese Vermutung liefern Johnson, Gächter und Herrmann (2005). Die Autoren berichten über eine Studie im Automobilmarkt, bei der Koeffizienten für die Verlustaversion von Probanden bezüglich vier PkwEigenschaften ermittelt wurden. Dabei zeigte sich, dass die Koeffizienten für den Benzinverbrauch und die Fahrzeugsicherheit (utilitaristische Produktfacetten) niedriger sind als für den Fahrkomfort und die Fahrerinformation (hedonistische Produktfacetten). Dieser Befund steht im Einklang mit den Erkenntnissen von Dhar und Wertenbroch (2000), die ebenfalls den Zusammenhang zwischen hedonistischen Gütern und der Verlustaversion betonen. Folglich dürfte es Individuen bei utilitaristischen Erzeugnissen leichter fallen, von einem Default (Vorselektion oder Empfehlung) abzuweichen und eine andere Option auszuwählen. Daher gilt folgende Hypothese:

H3: Bei hedonistischen Merkmalen wirkt der Default stärker als bei utilitaristischen auf die Absicht eines Individuums, die zum Default erhobene Merkmalsausprägung zu wählen.

II. Indirekte Wirkung von Defaults auf das Entscheidungsverhalten

Ohne Zweifel entscheiden sich Individuen in vielfältigen Kauf- und Konsumsituationen immer wieder gegen den Default. Dennoch ist vorstellbar, dass der Default selbst bei Entscheidungen gegen ihn eine zentrale Rolle bei der Produktwahlhandlung spielt. Den Erkenntnissen von McKenzie, Liersch und Finkelstein (2006) sowie McKenzie und Nelson (2003) zufolge zieht der Default die Aufmerksamkeit der Betrachter auf sich und wirkt in diesem Sinne als Referenzpunkt. Ausgehend vom Default betrachtet das Individuum alle weiteren Merkmalsausprägungen, wobei die in der Nähe des Referenzpunkts angesiedelten Ausprägungen zunächst evaluiert werden, bevor das Interesse den weiter entfernt präsentierten Optionen gilt (vgl. Chapman/Johnson, 1999, S. 118 ff.). Steht z. B. ein Kunde in einem Pkw-Konfigurator vor der Wahl eines Motors aus einer beachtlichen Zahl verschiedener Motorvarianten, ist folglich zu erwarten, dass die gewählte Option ,in der Nähe" des Default-Motors liegt. Hieraus ergibt sich folgende Hypothese:

H4: Die zum Default erhobene Merkmalsausprägung wirkt als Referenzpunkt, indem sie der Nachfrage nach Merkmalsausprägungen ,in ihrer Nähe“" zuträglich ist.

\section{Eine empirische Untersuchung}

\section{Anwendungsbereich}

Zur Überprüfung dieser Hypothesen bietet sich eine empirische Untersuchung unter Rückgriff auf einen Car-Konfigurator an. Aus praktischer Perspektive sind Konfiguratoren interessant, weil Unternehmen beachtliche Summen für ihre Gestaltung aufwenden. Insofern sind alle Erkenntnisse, die eine bessere Kundenorientierung ermöglichen, von Wichtigkeit. Aber auch aus theoretischer Sicht sind Konfiguratoren als Analysegegen- 
stand relevant, da die zuvor aufgeworfenen Entscheidungsprobleme bei einer online-Produktwahl nachgestellt werden können. Zudem finden sich Konfiguratoren nicht nur im Pkw-Sektor, sondern auch in anderen Branchen. Konfiguratoren kommen beispielsweise bei der Zusammenstellung von individualisierten Fonds oder bei der Gestaltung von Sportschuhen und Kosmetikartikeln zum Einsatz, so dass sich aus der empirischen Untersuchung für eine Vielzahl von Unternehmen bedeutsame Hinweise ableiten lassen.

Die Studie basiert auf einem Car-Konfigurator für eine bestimmte Baureihe mit allen seinen Varianten und Komponenten. Dieser online zugängliche Konfigurator wurde in allen seinen Verästelungen (d. h. mit allen Merkmalen und Ausprägungen) nachprogrammiert. Damit besteht die Möglichkeit, etwa die Art und Weise sowie die Reihenfolge der Präsentation der Merkmale und ihrer Ausprägungen nahezu beliebig zu verändern. Analog zum Original umfasst der nachprogrammierte Konfigurator 72 Merkmale wie Motor, Schalthebel, Lenkrad, Außenlackierung sowie Dekoreinlage, über deren Ausprägungen das Individuum entscheidet. Jedes Merkmal besteht aus mehreren Ausprägungen, wie etwa die Sitzeinstellung mit den Ausprägungen manuelle Höheneinstellung, elektrische Einstellung, elektrische Einstellung mit Memory-Funktion für Fahrersitz sowie elektrische Einstellung mit Memory-Funktion für beide Vordersitze.

\section{Experimentelles Design}

Zur Beantwortung der eingangs aufgeworfenen Fragen nach der Wirkung variierender Default-Stufen und -Quellen (Forschungsfragen 1 und 2) sowie der Bedeutung des Defaults als Referenz im Entscheidungsprozess (Forschungsfrage 3) kommt ein erstes in Tabelle 1 dargestelltes Design in Betracht, das aus drei verschiedenen Default-Stufen und vier verschiedenen Default-Quellen besteht. Die Variable „Default-Quelle“ umfasst die Vorselektion durch den Hersteller sowie die Empfehlungen dreier Testimonials. Aus Rücksicht auf die Anonymität des Kooperationspartners können diese Testimonials nicht benannt werden. Es lässt sich jedoch sagen, dass sie für unterschiedliche Kompetenzen stehen. Während man einer Person vor allem Wissen im Umgang mit der Fahrzeugtechnik attestiert, dürfte das Urteil einer anderen bei der Bewertung der Alltagstauglichkeit eines Pkw Gewicht besitzen. Dagegen zeichnet sich ein weiteres Testimonial durch seine Erfahrung im Motorsport aus. Zudem wurden die Default-Stufen „,niedrig“, „,mittel“ und „hoch“ berücksichtigt. Bei der Stufe „,niedrig“ (,mittel“, „,hoch“) ist der Default bei der im Preis niedrigsten (mittleren, höchsten) Ausprägung des jeweiligen Merkmals gesetzt.

Aus den drei Default-Stufen und den vier Default-Quellen lassen zwölf experimentelle Bedingungen (Konditionen) generieren. In jeder der zwölf Zellen befinden sich jeweils 72 Produktmerkmale mit ihren entsprechenden Ausprägungen. Sechs Merkmale wurden ausgewählt, um an ihnen die Default-Stufen und die Default-Quellen zu variieren. Hierzu gehören der Außenspiegel, das Lenkrad, der Motor, die Räder/Reifen, die Sitzbezüge und die Sitzeinstellung. Die Default-Quellen und -Stufen aller anderen 66 Merkmals blieben unverändert und wurden in der vom Hersteller präsentierten Art und Weise dargeboten.

Zur Erfassung der Vertrauenswürdigkeit des Absenders einer Empfehlung wurde eine Vorstudie durchgeführt. Hierbei forderte man 80 Probanden auf, die Vertrauenswürdigkeit der drei Testimonials im Rahmen der Wahl eines Fahrzeugs anzugeben. Im Sinne eines realistischen Untersuchungsdesigns wurden die Individuen in einem Einleitungs- 
Tab. 1. Beschreibung des Designs aus Default-Stufe und Default-Quelle

\begin{tabular}{|c|c|c|c|}
\hline \multirow[t]{2}{*}{ Default-Quelle } & \multicolumn{3}{|c|}{$\begin{array}{c}\text { Default-Stufe } \\
\text { für Außenspiegel, Lenkrad, Motor, Räder/Reifen, Sitzbezüge und } \\
\text { Sitzeinstellung }\end{array}$} \\
\hline & $\begin{array}{l}\text { Ausprägung mit } \\
\text { niedrigstem Preis }\end{array}$ & $\begin{array}{l}\text { Ausprägung mit } \\
\text { mittlerem Preis }\end{array}$ & $\begin{array}{l}\text { Ausprägung mit } \\
\text { höchstem Preis }\end{array}$ \\
\hline \multicolumn{4}{|l|}{ Vorselektion durch den Hersteller } \\
\hline \multicolumn{4}{|l|}{$\begin{array}{l}\text { Empfehlung durch ein Testimonial } \\
\text { mit Kompetenz bezüglich Fahrzeug- } \\
\text { technik }\end{array}$} \\
\hline \multicolumn{4}{|l|}{$\begin{array}{l}\text { Empfehlung durch ein Testimonial } \\
\text { mit Kompetenz bezüglich der } \\
\text { Alltagstauglichkeit von Pkw }\end{array}$} \\
\hline $\begin{array}{l}\text { Empfehlung durch ein Testimonial } \\
\text { mit Kompetenz bezüglich } \\
\text { Motorsport }\end{array}$ & & & \\
\hline
\end{tabular}

- Alle anderen 66 Merkmals wurde so präsentiert, wie sie auch vom Unternehmen im Markt dargeboten werden.

- Der Grad der Vertrauenswürdigkeit der drei Testimonials wurde in einem Pretest festgestellt.

text in die Situation der Fahrzeugwahl versetzt. Daraufhin bat man sie, ihre Urteile bezüglich der drei Absender von Empfehlungen auf einer 7er-Skala (1 = überhaupt keine Vertrauenswürdigkeit bis $7=$ sehr hohe Vertrauenswürdigkeit) abzugeben. Es zeigt sich, dass die Person mit Kompetenz zur Bewertung der Fahrzeugtechnik einen mittleren Wert von 3,84 (Standardabweichung = 1,39), jene mit Kompetenz im Motorsport einen von 3,19 $(1,32)$ und die mit Kompetenz zur Evaluation der Alltagstauglichkeit eines Pkw einen von $5,23(1,48)$ aufweist. Mittels einer Varianzanalyse und entsprechender Kontrastvergleiche lässt sich die statistische Signifikanz dieser Unterschiede verdeutlichen $(\mathrm{F}=44,163 ; \mathrm{p}=$ 0,000; Differenz „Kompetenz Fahrzeugtechnik“ gegenüber „Kompetenz Motorsport“ = 0,65; $\mathrm{p}=0,004$; Differenz „Kompetenz Fahrzeugtechnik“ gegenüber „Kompetenz Alltagstauglichkeit" $=-1,39 ; \mathrm{p}=0,000)$.

Darüber hinaus ist ein zweites Untersuchungsdesign zu erstellen, mit dem sich die Frage nach der verhaltensprägenden Wirkung des Defaults bei utilitaristisch bzw. hedonistisch präsentierten Produktmerkmalen beantworten lässt (Forschungsfrage 4). Hierzu kommt ein experimentelles Design in Betracht, das die Merkmale Audio, Beleuchtung, Fahrhilfe, Kommunikation, Parksystem, Scheinwerfer, Transport und Verbrauch umfasst. Im Sinne einer handhabbaren Datenerfassung besteht jedes Merkmal lediglich aus drei Ausprägungen, die in Tabelle 2 dargestellt sind. Für das Merkmal Audio existiert eine utilitaristische und eine hedonistische Variante der Merkmalspräsentation, wobei erstere den realen Gegebenheiten entspricht und letztere erfunden ist. Die sieben anderen Merkmale wurden hingegen lediglich in der utilitaristischen Variante dargeboten. Bei drei Default-Stufen (Preis niedrig, mittel, hoch) und zwei Spielarten zur Darbietung des Merkmals Audio (utilitaristisch bzw. hedonistisch) ergeben sich insgesamt sechs Konditionen. Beispielsweise sind in Kondition 1 bei allen Merkmalen die Defaults auf die Ausprägungen mit dem niedrigsten Preis gesetzt, und Audio ist als utilitaristisches 


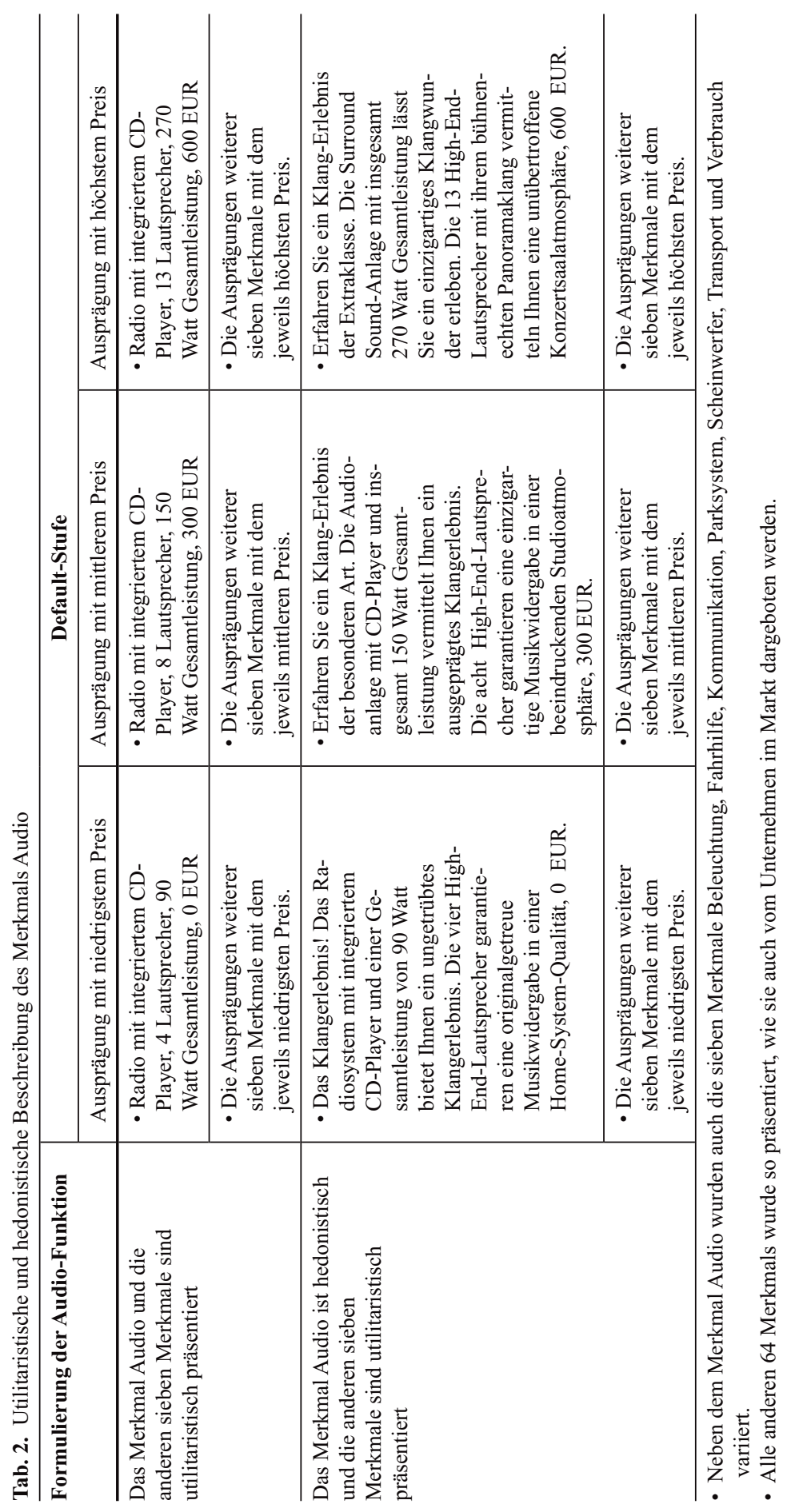


Merkmal dargeboten. In Kondition sechs entsprechen die Defaults bei allen Merkmalen den im Preis höchsten Ausprägungen, und Audio ist in der hedonistischen Variante präsentiert.

\section{Abhängige Variablen}

Als abhängige Größe fungierte die Preisausschöpfung. Sie gibt das Ausmaß an, in dem die Individuen, die mit einer bestimmten Kombination aus Default-Stufe und DefaultQuelle konfrontiert wurden, das Preisspektrum bei den sechs Merkmalen ausschöpften. Hätten die Probanden bei allen Merkmalen lediglich die Standardoption (geringwertigste Merkmalsausprägung ohne Aufpreis) gewählt, würde die Preisausschöpfung 0 Prozent lauten. Hingegen betrüge dieser Wert 100 Prozent, würden die Personen bei allen Merkmalen jeweils die teuerste Ausprägung (für die ein entsprechender Aufpreis zu bezahlen ist) selektieren. Eine weitere abhängige Variable bildet die Häufigkeit, mit der die Probanden den Default wählen. Hierbei geht es sowohl um die Anzahl der Entscheidung für bzw. gegen den Default als auch um das Verhältnis dieser beiden Werte.

\section{Ablauf}

Zur Durchführung der empirischen Untersuchung ist es erforderlich, den replizierten Konfigurator entsprechend den erläuterten Konditionen zu verändern. Hierbei wurde für jede Kondition eine Konfigurator-Variante programmiert. Alle von der jeweiligen Manipulation nicht berührten Merkmale erschienen in der im Originalkonfigurator präsentierten Art und Weise. Um die Aussagekraft der Studie zu gewährleisten, verknüpfte man die Konfigurator-Varianten mit der Homepage der betrachteten Pkw-Baureihe. Jeder zwanzigste Besucher dieser Site wurde eingeladen, an der empirischen Untersuchung teilzunehmen. Im Falle seiner Zusage wurde er einer Kondition zugeordnet und durchlief die entsprechende Konfiguration. Alle für die Studie relevanten Variablen konnten automatisch (d. h. ohne weitere Angaben der Probanden) erfasst werden. Aus dem Entscheidungsverhalten (den einzelnen Klicks) eines Individuums ergaben sich merkmalspezifische Informationen über die Wahl des jeweiligen Defaults oder einer anderen Merkmalsausprägung. Dazu konnte der Preis für die gewählte Ausprägung ermittelt und am Ende des Konfigurationsprozesses der Gesamtpreis für das spezifizierte Fahrzeug errechnet werden.

Aufgrund rechtlicher Restriktionen ist es derzeit für Endkunden (im Unterschied zu Flottenkunden und Mietwagengesellschaften) noch nicht möglich, ein Fahrzeug aus dem Car-Konfigurator heraus zu bestellen. Es besteht jedoch die Option, das spezifizierte Fahrzeug zum Pkw-Händler zu mailen und dort die Bestellung auszulösen. Insofern können in der Studie allenfalls Präferenzen und keine tatsächlichen Umsätze erfasst werden. Da uns jedoch Daten vorliegen, die eine beachtliche Deckungsgleichheit zwischen den im Konfigurator spezifizierten Fahrzeugen und den tatsächlich bestellten zeigen, soll die Umsatzkalkulation beibehalten werden, wohl wissend, dass es nicht um realisierte Umsätze, sondern bestenfalls um Umsatzpotenziale geht. 


\section{Ergebnisse aus Experiment 1}

Um die Wirkung der Default-Stufe und der Default-Quelle (unabhängige Variablen) auf das Entscheidungsverhalten der Individuen zu testen (Forschungsfragen 1 und 2), bietet es sich an, unter Rückgriff auf die Stimulimenge (Außenspiegel, Lenkrad, Motor, Räder/ Reifen, Sitzbezüge und Sitzeinstellung) zwölf verschiedene Gruppen von Probanden mit unterschiedlichen Stimuli zu konfrontieren (vgl. Tabelle 1). Die Mitglieder jeder Gruppe durchliefen den Car-Konfigurator, der aus den 66 nicht veränderten Merkmalen und den sechs zellenspezifisch angepassten Merkmalen bestand.

Ein Blick auf Tabelle 3 zeigt, dass die Preisausschöpfung (PA) bei der niedrigsten Default-Stufe unabhängig von der Default-Quelle gering ist. Dagegen lässt sich durch die höchste Default-Stufe (wiederum unabhängig von der Default-Quelle) eine beachtliche Preisausschöpfung realisieren. Ein Blick auf die in Tabelle 3 dargebotenen Tests verdeutlicht, dass sich Hypothese H1 vorläufig bestätigen lässt. Auch über die Default-Quellen ist Variation zu beobachten; die Empfehlung des Testimonials mit Kompetenz bezüglich Motorsport und die Vorselektion durch den Hersteller sind weniger geeignet, damit Kunden das Aufpreisspektrum ausschöpfen. Im Unterschied dazu erweisen sich die Empfehlungen der Testimonials mit Kompetenz bezüglich der Alltagstauglichkeit von Pkw und der Fahrzeugtechnik als besser geeignet, um Kunden zur Wahl einer aufpreispflichtigen Option zu bewegen.

Anhand der Vorselektion durch den Hersteller und der Empfehlung des Testimonials mit Kompetenz bezüglich der Fahrzeugtechnik lässt sich das Entscheidungsverhalten verdeutlichen: Folgten alle Kunden dem Default, wäre die Aufpreisausschöpfung beim niedrigen Default 0 Prozent und beim hohen Default 100 Prozent. Tabelle 3 zeigt, dass insbesondere die Vorselektion durch den Hersteller in Kombination mit einem niedrigen Default besonders viele Individuen veranlasst, den Default zu wählen. Dagegen geht von der Empfehlung des Testimonials mit Kompetenz bezüglich der Fahrzeugtechnik bei einem hohen Default ein besonderer Effekt auf die Absicht der Kunden aus, sich für den Default zu entscheiden.

Offenbar hängt die Wirkung der Default-Stufe (Default-Quelle) auf das Entscheidungsverhalten vom Niveau der Default-Quelle (der Default-Stufe) ab. Dieser Interaktionseffekt tritt besonders deutlich in einer Veränderung der Default-Stufe von mittel zu hoch zu Tage. Kommt die Empfehlung des Testimonials mit Kompetenz bezüglich Motorsport zum Einsatz, ist kein merklicher Effekt (von 31,4\% auf 30,3\%) zu konstatieren, wohingegen die Empfehlung des Testimonials mit Kompetenz bezüglich der Fahrzeugtechnik als Default eine beachtliche Steigerung der Aufpreisausschöpfung (von 29,1\% auf $45,9 \%$ ) bewirkt.

Darüber hinaus interessiert die Relevanz von Vorgaben und Empfehlungen für die Bereitschaft der Kunden, sich für den Default zu entscheiden. Zu diesem Zweck wurde die prozentuale Häufigkeit ermittelt, mit der die Kunden den Default in Abhängigkeit unterschiedlicher Default-Quellen wählten. Es ist zu erkennen, dass bei einer Vorselektion in 34,8\% der Entscheidungen die Wahl auf den Default fiel. Bildete hingegen die Empfehlung des Testimonials mit Kompetenz bezüglich Alltagstauglichkeit (Fahrzeugtechnik bzw. Motorsport) den Default, lautete der entsprechende Wert 24,7\%, 19,0\% bzw. 13,8\%. Ein Blick auf die z-Werte eines ,sample proportion“-Tests liefert folgende Erkenntnis: 
Tab 3. Ergebnisse der ANOVA zur Preisauschöpfung

\begin{tabular}{l|c|c|c}
\hline \multirow{2}{*}{ Default-Quelle } & \multicolumn{3}{|c}{$\begin{array}{c}\text { Default-Stufe } \\
\text { fitzeinstellung }\end{array}$} \\
\cline { 2 - 4 } & $\begin{array}{c}\text { Ausprägung mit } \\
\text { niedrigstem Preis }\end{array}$ & $\begin{array}{c}\text { Ausprägung mit } \\
\text { mittlerem Preis }\end{array}$ & $\begin{array}{c}\text { Ausprägung mit } \\
\text { höchstem Preis }\end{array}$ \\
\hline Vorselektion durch den Her- & $\mathrm{PA}=14,5$ & $\mathrm{PA}=32,4$ & $\mathrm{PA}=39,5$ \\
steller & $(\mathrm{s}=19,1)$ & $(\mathrm{s}=31,5)$ & $(\mathrm{s}=34,2)$ \\
& $(\mathrm{N}=55)$ & $(\mathrm{N}=49)$ & $(\mathrm{N}=46)$ \\
\hline Empfehlung durch ein & $\mathrm{PA}=23,4$ & $\mathrm{PA}=29,1$ & $\mathrm{PA}=45,9$ \\
Testimonial mit Kompetenz & $(\mathrm{s}=36,8)$ & $(\mathrm{s}=33,8)$ & $(\mathrm{s}=43,4)$ \\
bzg. Fahrzeugtechnik & $(\mathrm{N}=50)$ & $(\mathrm{N}=52)$ & $(\mathrm{N}=53)$ \\
\hline Empfehlung durch ein Testimo- & $\mathrm{PA}=29,0$ & $\mathrm{PA}=32,9$ & $\mathrm{PA}=39,6$ \\
nial mit Kompetenz bzg. der & $(\mathrm{s}=33,3)$ & $(\mathrm{s}=36,9)$ & $(\mathrm{s}=40,6)$ \\
Alltagstauglichkeit von Pkw & $(\mathrm{N}=52)$ & $(\mathrm{N}=52)$ & $(\mathrm{N}=55)$ \\
\hline Empfehlung durch ein & $\mathrm{PA}=15,4$ & $\mathrm{PA}=31,4$ & $\mathrm{PA}=30,3$ \\
Testimonial mit Kompetenz & $(\mathrm{s}=29,8)$ & $(\mathrm{s}=36,8)$ & $(\mathrm{s}=37,1)$ \\
bzg. Motorsport & $(\mathrm{N}=49)$ & $(\mathrm{N}=55)$ & $(\mathrm{N}=50)$ \\
\hline
\end{tabular}

$\mathrm{PA}=$ durchschnittliche Preisausschöpfung in \%

$\mathrm{s}=$ Standardabweichung

$\mathrm{N}=$ Anzahl Probanden pro Kondition

ANOVA

Default-Stufe: $F=31,980, p=0,000$

Default-Quelle: $F=5,330, p=0,001$

Interaktion (Default-Stufe, Default-Quelle): $\mathrm{F}=2,526, \mathrm{p}=0,020$

Vgl. zu den verwendeten Tests etwa Backhaus et al., 2003, Hair et al., 1998

Der Unterschied in der Default-Wahlhäufigkeit zwischen der Vorselektion und der jeweiligen Empfehlung ist statistisch signifikant verschieden von Null (z-Wert: -2,669, -4,293 bzw. -4,507; der kritische Wert lautet bei einer einprozentigen Irrtumswahrscheinlichkeit -2,325). Demnach wirkt ein harter Default stärker auf das Entscheidungsverhalten der Individuen als ein weicher, so dass Hypothese $2 \mathrm{a}$ vorläufig zu bestätigen ist.

Zudem zeigt diese Analyse, dass auch zwischen den Empfehlungen Unterschiede im Hinblick auf deren Wirkung auf das Entscheidungsverhalten bestehen. Die Empfehlung des Testimonials mit der Kompetenz bezüglich Alltagstauglichkeit übt einen größeren Effekt auf die Bereitschaft der Individuen zur Wahl eines Defaults aus als der Ratschlag aus der Quelle mit Kompetenz hinsichtlich Fahrzeugtechnik $(\mathrm{p}<0,1)$, der seinerseits einen stärkeren Einfluss entfaltet als die Empfehlung jener Person, die für Kompetenz im Motorsport steht $(\mathrm{p}<0,1)$. Der Unterschied zwischen der Defaultwahlhäufigkeit bei einer Empfehlung des Individuums mit der Kompetenz „Alltagstauglichkeit“ unterscheidet sich vom entsprechenden Wert bei einer Empfehlung auf Basis der Kompetenz „Motorsport" deutlich $(\mathrm{p}<0,01)$.

Hält man sich diese Rangfolge der Vertrauenswürdigkeit der Absender von Empfehlungen vor Augen (Kompetenz „Alltagstauglichkeit“ gefolgt vom Kompetenz „Technik“ gefolgt vom Kompetenz „Motorsport“, vgl. dazu die in Kapitel D.II erläuterte Vorstudie) und vergleicht diese mit der Bedeutung der drei Quellen für die Bereitschaft der Kunden, 
den Default zu wählen, ergibt sich folgendes Bild: Die besonders vertrauenswürdige Quelle „Kompentenz Alltagserfahrung“ vermag sehr viele Probanden zur Wahl des Defaults zu überzeugen. Dagegen fällt es dem als am wenigsten vertrauenswürdig eingestuften Testimonial mit der Kompetenz „Motorsport“ auch deutlich schwerer, Kunden zur Wahl des Defaults zu veranlassen. Die Person mit der Kompetenz bezüglich Fahrzeugtechnik liegt im Hinblick auf ihre Vertrauenswürdigkeit und ihre Macht, die Wahl eines Defaults zu suggerieren, zwischen den beiden anderen Absendern von Empfehlungen. Insofern gilt Hypothese $\mathrm{H} 2 \mathrm{~b}$ als vorläufig bestätigt.

Zuvor war die Rede davon, dass sich Individuen immer wieder gegen Vorgaben und Empfehlungen entscheiden. Gleichwohl fungieren Defaults als Referenzpunkte, die den Absatz von Merkmalsausprägungen ,,in ihrer Nähe“ fördern (Forschungsfrage 3). Um diesen Effekt zu untersuchen, soll nicht nur die Anzahl der Entscheidung für bzw. gegen den Default betrachtet werden. Vielmehr interessiert die Verteilung der Häufigkeit, mit der die Probanden die einzelnen Merkmalsausprägungen wählen, in Abhängigkeit variierender Defaults. Zu diesem Zweck zeigt Abbildung 1 bei verschiedenen Default-Stufen (niedriger Preis, mittlerer Preis, hoher Preis) die Häufigkeit, mit der die Ausprägungen des Merkmals „Lenkrad“ von den Individuen selektiert wurden. Die andere Dimension bringt das Preisspektrum der Ausprägungen zum Ausdruck; während bei 0 Euro das aufpreisfreie Standardlenkrad angesiedelt ist, befindet sich das teuerste frei wählbare Lenkrad bei 950 Euro. Zwischen diesen Polen sind alle anderen Lenkrad-Optionen entsprechend ihrem Aufpreis zu finden. Allerdings existiert nicht bei jedem Punkt auf dem Kontinuum der Aufpreisausschöpfung eine Option, und nicht jedes verfügbare Lenkrad wurde von den Individuen in der Studie tatsächlich gewählt.

Ein Blick auf Abbildung 1 verdeutlicht, dass sich der Verlauf der Häufigkeitsverteilung in Abhängigkeit des Defaults verändert. Während die Kunden bei niedrigem Default (linkes Schaubild) vor allem Merkmalsausprägungen wünschen, die zwischen der geringwertigsten und der mittleren Lenkrad-Option angesiedelt sind, interessieren bei mittlerem Default vor allem Optionen „links und rechts“ dieses Ankerpunkts (mittleres Schaubild). Diese Verschiebung des Defaults führt zu einem beachtlichen Rückgang der Nachfrage nach geringwertigen Lenkrädern, wohingegen die Merkmalsausprägungen im mittleren Bereich des Ausprägungskontinuums an Bedeutung gewinnen. Eine nochmalige Verschiebung des Defaults auf das hochwertigste Lenkrad bewirkt einen Anstieg der Nachfrage nach eben dieser hochwertigen Option (rechtes Schaubild). Außerdem fällt die Nachfrage nach dem geringwertigsten Lenkrad weiter, und die mittleren Optionen gewinnen an Bedeutung.

Dem Kolmogorov-Smirnov-Test zufolge weichen beim Lenkrad (und beim Motor) die Häufigkeitsverteilungen im linken und mittleren sowie im linken und rechten Schaubild statistisch signifikant voneinander ab (für Lenkrad: Default niedrig vs. Default mittel: $p$ $=0,006$; Default mittel vs. Default hoch: $\mathrm{p}=0,152$; Default niedrig vs. Default hoch: $\mathrm{p}=$ 0,001). Dagegen unterscheiden sich die durch den mittleren und hohen Default bewirkten Häufigkeitsverteilungen nicht deutlich. Bei den anderen Merkmalen zeigt sich ein gemischtes Bild; während bei den Räder/Reifen und dem Außenspiegel Unterschiede zwischen den einzelnen Verteilungen auftreten, sind bei den Sitzbezügen und der Sitzeinstellung lediglich Abweichungen zwischen Verteilungen bei niedrigem und hohem Default festzustellen. Folglich kann die Bedeutung von Defaults als Referenzpunkte für die Aus- 

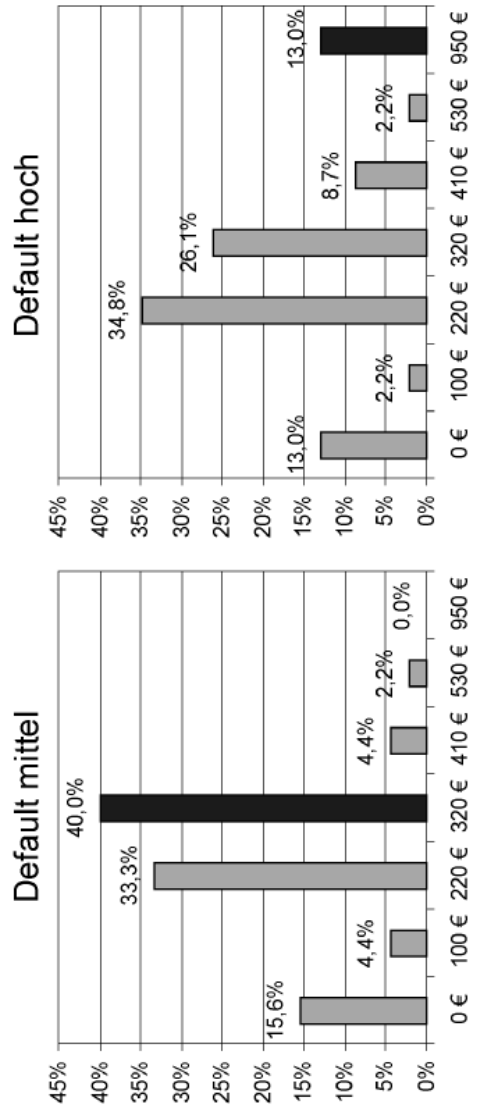

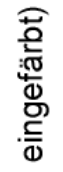

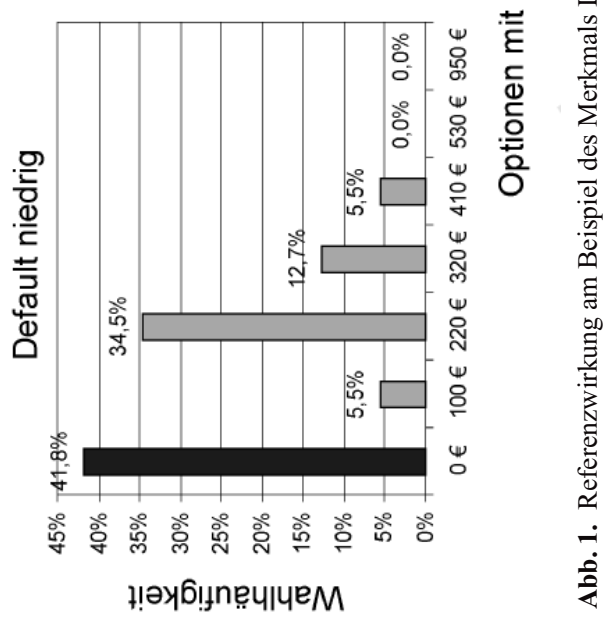


wahl von Merkmalsausprägungen grundsätzlich postuliert werden (vgl. Hypothese 4). Jedoch bestehen merkmalspezifische Effekte, die im Rahmen dieser Studie nicht analysiert werden konnten und insofern einer separaten Betrachtung bedürfen.

\section{Ergebnisse aus Experiment 2}

Zur Analyse der Wirkung des Defaults bei utilitaristischen und hedonistischen Merkmalen dienen die in Tabelle 2 präsentierten Merkmale und deren Ausprägungen (Forschungsfrage 4). Wie erinnerlich besteht jedes Merkmal aus drei (utilitaristischen) Ausprägungen, und für das Produktmerkmal Audio existiert eine utilitaristische und eine hedonistische Spielart der Merkmalsdarbietung (drei utilitaristische und drei hedonistische Ausprägungen). Aus diesem Untersuchungsdesign ergeben sich sechs Konditionen, denen jeweils 75 Probanden zugewiesen wurden. Da jedes Individuum über acht Merkmale entscheidet, liegen (sofern jeder Proband bei jedem Merkmal eine Wahl trifft) in jeder Kondition 600 Entscheidungen über ausgewählte Merkmalsausprägungen vor (75 Personen mal 8 Entscheidungen).

Aus Tabelle 4 geht die Anzahl der für bzw. gegen den Default getroffenen Entscheidungen in Abhängigkeit der Kondition hervor. Beispielsweise entschieden sich die Individuen in Kondition 1 (Preisniveau niedrig, Audio ist utilitaristisch) nur 156 mal für den Default und 340 mal für eine andere Merkmalsausprägung. Dagegen wählten die Kondition 6 (Preisniveau hoch, Audio ist hedonistisch) zugeordneten Probanden 300 mal den Default und lediglich 148 mal eine alternative Option. Neben diesen aggregierten, d. h. über alle Merkmale betrachteten Häufigkeitswerte, liefert Tabelle 4 zudem Angaben über die Anzahl der Entscheidungen für und gegen den Default allein beim Merkmal „Audio“.

Es fällt auf, dass sich im aggregierten Fall (d. h. die Entscheidungen über alle Merkmale) das Verhältnis der Anzahl Entscheidungen für und gegen den Default über die sechs Konditionen verändert. Analog dazu lässt sich auch für den disaggregierten Fall (d. h. allein die Entscheidungen bezüglich des Merkmals Audio) eine statistisch signifikante Wirkung der Kondition auf die Wahl des Defaults bzw. einer anderen Option konstatieren. Eine ergänzende Analyse zeigt, dass von den über die Individuen variierenden Präferenzen für die einzelnen Merkmalsausprägungen kein statistisch signifikanter Effekt auf die Anzahl der Entscheidungen für bzw. gegen den Default ausgeht. Wäre dies der Fall, müsste ein Teil der Varianz in den Daten dem Wirken individueller Präferenzen und nicht allein nur den Konditionen zugeschrieben werden.

Aus den vorliegenden Zahlen ist zu erkennen, dass die Anzahl gewählter Defaults (sofern der Default auf ein niedriges Preisniveau gesetzt ist) bei einem hedonistischen Merkmal niedriger ist als bei einem utilitaristischen. Dieser Befund dreht sich bei einem höheren Preisniveau für den Default um; in diesem Fall wählen die Individuen bei hedonistischen Merkmalen häufiger den Default als bei utilitaristischen. Im Hinblick auf die Überprüfung von Hypothese 3 liegt ein gemischtes Bild vor. In der Tat wirkt der Default bei hedonistischen Merkmalen stärker als bei utilitaristischen, allerdings nur dann, wenn der Default selbst am oberen Ende des Ausprägungsspektrums angesiedelt ist. Setzt man den Default hingegen am unteren Ende des Kontinuums der Merkmalsausprägungen, ent- 


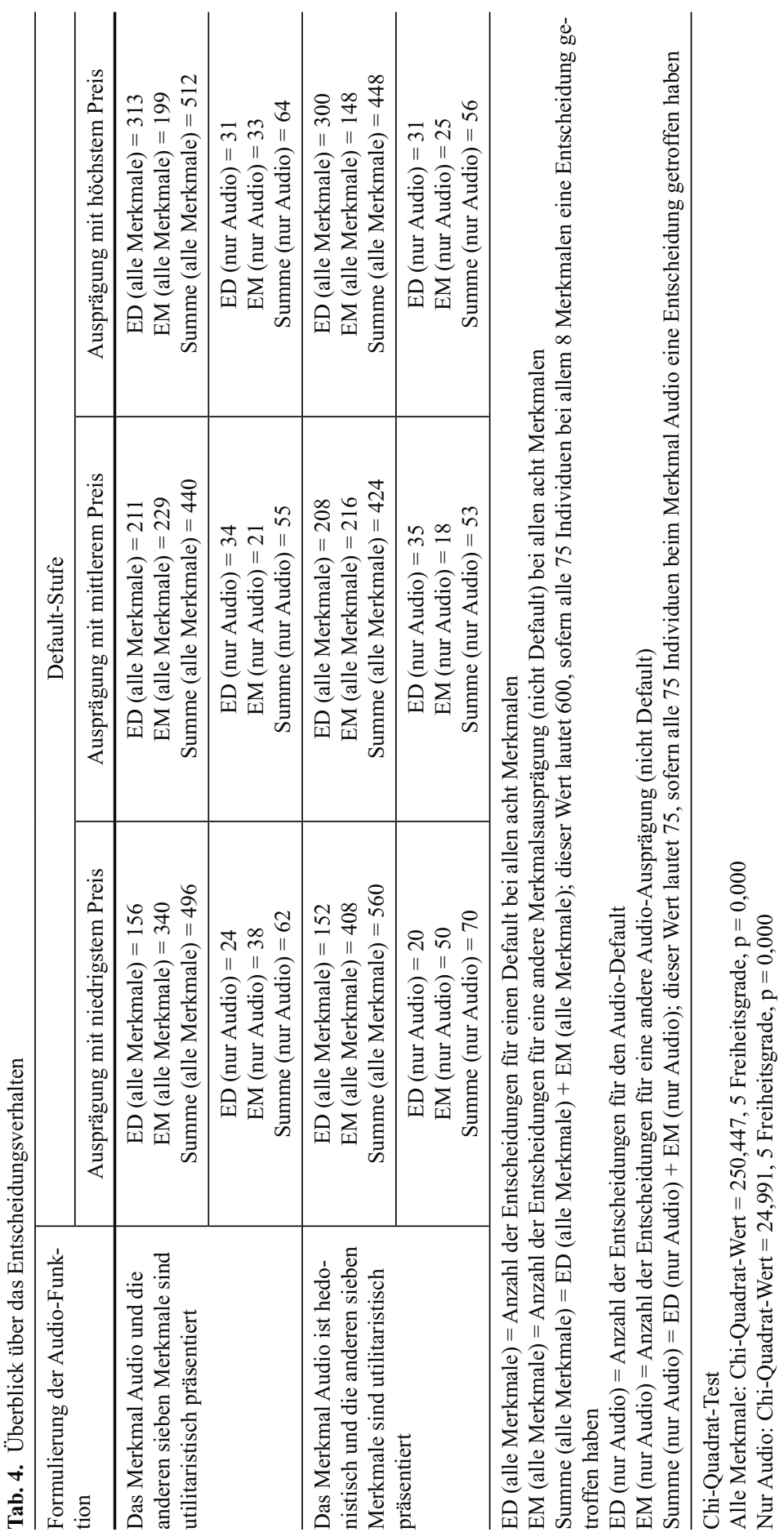


faltet er bei utilitaristischen Merkmalen einen größeren Effekt auf das Entscheidungsverhalten als bei hedonistischen. Damit ist Hypothese 3 teilweise bestätigt.

\section{Implikationen für Theorie und Praxis}

Die in Wissenschaft und Praxis geführte Diskussion über die Gestaltung von Vorgaben und Empfehlungen basiert auf dem Befund, dass sie grundsätzlich einen Einfluss auf das Entscheidungsverhalten ausüben. Allerdings sind wichtige Fragen etwa nach dem Effekt unterschiedlicher Default-Varianten noch nicht beantwortet. Die im Rahmen der Studien erzielten Ergebnisse ermöglichen eine differenzierte Betrachtung des Wirkungszusammenhangs zwischen der Beschaffenheit von Defaults (entweder als Vorgabe oder als Empfehlung) und dem Entscheidungsverhalten der Individuen. Aus den gewonnenen Erkenntnissen ergeben sich konkrete Anhaltspunkte für die Gestaltung z. B. von onlineKonfiguratoren, von Formularen zur Spezifikation von Produkten und Diensten sowie von Empfehlungen, die im persönlichen Gespräch oder im Rahmen einer Massenkommunikation abgegeben werden.

\section{Forschungsfrage 1: Wirkung der Default-Stufe auf das Entscheidungsverhalten}

Es konnte gezeigt werden, dass eine Verschiebung des Defaults bei einem Merkmal (z. B. Scheinwerfer) von der geringwertigsten (Halogenscheinwerfer) zu höherwertigen Merkmalsausprägungen (Xenon-Scheinwerfer) zu einer Steigerung des (potenziellen) Umsatzes führt. Überraschenderweise sind in nahezu allen Produktkonfiguratoren die merkmalspezifischen Defaults bei der geringwertigsten Merkmalsausprägung gesetzt. Allerdings sollte die Verschiebung des Defaults zu einer höherwertigen Option zumindest schrittweise erfolgen, und es ist zu bedenken, inwieweit besonders hochwertige Merkmalsausprägungen überhaupt als Default in Betracht kommen. Die Ausführungen zum Konzept der „,marketplace metacognition“ haben gezeigt, dass Kunden Überlegungen anstellen, ob der Default in ihrem oder im Interesse des Unternehmens gesetzt ist. Gelangen sie zu dem Urteil, der Default sei allein deshalb definiert worden, um den Umsatz bzw. Gewinn des Unternehmens zu optimieren, entsteht Reaktanz gegenüber der Vorgabe oder Empfehlung (vgl. Obermiller/Spangenberg, 1999, S. 163 ff.).

\section{Forschungsfrage 2: Wirkung der Default-Quelle auf das Entscheidungsverhalten}

Aus der Untersuchung folgt zudem, dass ein harter Default (eine Vorgabe) stärker auf das Entscheidungsverhalten der Individuen wirkt als ein weicher Default (eine Empfehlung). Zudem hängt die Wirkung der weichen Defaults auf das Entscheidungsverhalten von der Vertrauenswürdigkeit des Absenders der Empfehlung ab (vgl. Ahearne/Gruen/Jarvis, 1999). Will man Individuen zur Wahl des Defaults stimulieren, sollten Vorgaben gemacht werden, etwa durch Voreinstellungen im online-Konfigurator oder durch eine Vorauswahl von Merkmalsausprägungen in einem Bestell- bzw. Anmeldeformular (vgl. McFarland/ Challagalla/Shervani, 2006). Während nahezu alle online-Konfiguratoren aufgrund spezifikationstechnischer Notwendigkeiten solche Voreinstellungen aufweisen (z. B. müssen 
für ein Fahrzeug zwingend Motor, Felgen und andere Merkmale ausgewählt werden), zeigt eine Analyse der Formulargestaltung kein einheitliches Bild. Einige Banken und Versicherungen nehmen gezielt durch eine Vorauswahl von Merkmalsausprägungen in Formularen zur Spezifikation einer Kapitalanlage oder eines Versicherungsvertrags auf das Entscheidungsverhalten ihrer Kunden Einfluss. Dagegen kommen Defaults bei vielen anderen Unternehmen beispielsweise im Tourismussektor zur Festlegung einer Urlaubsreise oder in der Telekommunikationsbranche bei der Ausgestaltung eines Handy-Vertrags bislang nur in seltenen Fällen zum Einsatz.

\section{Forschungsfrage 3: Wirkung des Defaults als Referenzpunkt auf das Entschei- dungsverhalten}

Hier konnte Aufschluss darüber erzielt werden, dass die zum Default erhobene Merkmalsausprägung als Referenzpunkt wirkt, indem sie dem Absatz von Merkmalsausprägungen ,in ihrer Nähe“ zuträglich ist. Defaults ziehen Nachfrage ,in ihre Nähe“ und üben offenbar selbst dann Einfluss auf das Entscheidungsverhalten aus, wenn sie von den Kunden nicht ausgewählt werden. Dieser Befund deutet auf einen beachtlichen indirekten (neben dem berichteten direkten) Effekt von Defaults auf das Entscheidungsverhalten hin.

\section{Forschungsfrage 4: Wirkung des Defaults bei utilitaristischen und hedonistischen Merkmals auf das Entscheidungsverhalten}

Diese Analyse förderte die Erkenntnis zutage, dass bei hedonistischen Merkmalen der Default stärker wirkt als bei utilitaristischen Merkmalen, allerdings nur dann, wenn der Default am oberen Ende des Ausprägungsspektrums angesiedelt ist. Bildet hingegen die Ausprägung am unteren Ende den Default, ist die Wirkung bei utilitaristischen Optionen stärker. Folglich bietet es sich an (zumindest bei jenen Merkmalen, die in ihrem Wesen hedonistisch darstellbar sind), die zumeist dominierende utilitaristische Art und Weise ihrer Präsentation zu überdenken und eine hedonistische Variante in Zusammenhang mit einem im Preis hohen Default zu bilden.

Hält man sich die Erkenntnisse vor Augen, ist die Vorstellung zu korrigieren, Kunden würden über ein umfassendes Gefüge verankerter Präferenzen verfügen, die sie invariant gegenüber den jeweiligen Entscheidungstatbeständen im Konfigurator machen. Im Gegenteil, eine Reihe vermeintlich unbedeutender Charakteristika von Defaults determiniert das Entscheidungsverhalten (vgl. Heitmann/Herrmann, 2006). Dieser Befund ist insofern von Bedeutung, als immer mehr Unternehmen es dem Kunden überlassen, ein Produkt nach seinen Wünschen und Vorstellungen zu gestalten. Hierzu dienen Konfiguratoren oder auch ,toolkits“, die inzwischen in nahezu allen Industrien verbreitet sind.

Obgleich das Augenmerk in diesem Beitrag vor allem der kundenorientierten Gestaltung eines Car-Konfigurators galt, sind die gewonnenen Erkenntnisse auch für Unternehmen in anderen Wirtschaftszweigen relevant. Man denke etwa an Banken und Versicherungen, bei denen ein Kunde häufig mittels Fragebogen (was einer offline-Konfiguration entspricht) eine Kraftfahrzeugversicherung oder eine Immobilienfinanzierung spezifiziert. Weitere Beispiele für Produktkonfigurationen jedweder Art, die der Kunde selbst 
vornimmt, finden sich in der PC-Branche, im Reisesektor oder in der Sportartikelindustrie. Ohne Zweifel ist mit einer weiteren Diffusion solcher Konfiguratoren zu rechnen, so dass die Frage nach der kundengerechten Gestaltung von vielen Unternehmen zu beantworten ist.

Obwohl die deskriptive Entscheidungstheorie Erkenntnisse für die Konfiguratorengestaltung liefert, werfen solche Technologien spezifische Fragen auf, die bislang noch unerforscht sind. Hersteller führen oft Zwangskopplungen von einzelnen Merkmalen ein, um die Komplexität der Mass Customization zu kontrollieren. Ein Beispiel dafür ist ein Automobilhersteller; will ein Kunde ein Fahrzeug mit Ledersitzen bestellen, muss gleichzeitig ein aufpreispflichtiges Lederlenkrad erworben werden. Kunden, die sich für Ledersitze, aber nicht für ein Lederlenkrad interessieren, stehen nach der ersten Entscheidung für die Bepolsterung vor dem Problem, eine relative Gewichtung zwischen Sitzen und Lenkrädern vornehmen zu müssen, da Entscheidungen zwischen vergleichbaren Sitzbezügen und nicht-vergleichbaren Lenkrädern und Sitzen sich gegenseitig bedingen. Die Konsequenzen einer derartigen Konstellation für das Entscheidungsverhalten und damit auch für die Gestaltung von Defaults sind unklar.

Ein anderes Problem betrifft die Möglichkeit von Individuen, in Konfiguratoren einmal getroffene Entscheidungen umzukehren. Im Gegensatz zur physischen Welt kann der Nutzer im Konfigurator stets die vorangegangene Auswahl rückgängig machen und verändern. Befürchten Kunden Nachkauf-Regret, ist zu erwarten, dass sie sich nicht auf eine Alternative festlegen wollen, sondern ihre Auswahl wiederholt überdenken (vgl. Zeelenberg, 1999). Im Gegensatz dazu wäre es ebenfalls plausibel, würden Individuen an ihren einmal konstruierten Präferenzen festhalten und abgeneigt sein, Konfigurationen nachträglich zu verändern (vgl. Wallsten/Barton, 1982). In diesem Zusammenhang ist zu bedenken, dass Nutzer am Heimcomputer nach der fertigen Konfiguration (noch) keine unmittelbare Bestellung auslösen können, sondern ihre Einstellung speichern, drucken, notieren oder zum Händler mailen. Bislang ist unbekannt, wie Individuen, deren Produktauswahl durch den Entscheidungskontext geprägt wurde, später auf ihre eigene Konfiguration reagieren. So ist vorstellbar, dass Individuen zwar unmittelbar nach der Produktanpassung mit ihrer Auswahl zufrieden sind, jedoch bei späteren, erneuten Prüfungen, Inkonsistenzen zwischen ihren Bedürfnissen und ihrer Konfiguration bemerken und vom Produkterwerb absehen.

In diesem Zusammenhang spielt auch der Einfluss von Händlern eine Rolle. Neben Vorgaben, die im Konfigurator gesetzt sind, geben Händler Empfehlungen ab, die sich auf das Entscheidungsverhalten der Individuen auswirken. Folglich steht der Kunde in vielen Entscheidungssituationen einer Kombination von harten und weichen Defaults gegenüber. Erkenntnisse über den Einfluss mehrerer Defaults liegen bislang noch nicht vor.

Abschließend soll noch auf das bereits aufgeworfene Phänomen der Interaktion zwischen Merkmal, Default-Stufe und Default-Quelle eingegangen werden. Eine umfassende Analyse des Zusammenhangs zwischen diesen Variablen war in Anbetracht der Komplexität des dafür notwendigen Untersuchungsdesigns nicht möglich. Jedoch lassen sich zwei Trends erkennen, deren tiefergehende Untersuchung vielversprechend erscheint: Bei Merkmalen, die technisch anmuten und erklärungsbedürftig sind (z. B. Einparkhilfe), ist die Bereitschaft der Kunden besonders groß, den Default zu wählen. Ein ähnlicher 
Effekt ist bei Merkmalen zu erkennen, die die aktive und passive Sicherheit betreffen. Besitzen die Individuen hingegen Wissen und Erfahrungen über ein Merkmal (möglicherweise aus anderen Lebensbereichen), wie etwa bei der Wahl von Sitzbezügen, entscheiden sie sich eher gegen den Default.

\section{Literatur}

Ahearne, M./Gruen, T. W./Jarvis, C. B. (1999): If Looks Could Sell: Moderation and Mediation of the Attractiveness Effect on Salesperson Performance, in: International Journal of Research in Marketing, Vol. 16, S. 269-284.

Backhaus, K./Erichson, B./Plinke, W./Weiber, R. (2003): Multivariate Analysemethoden, 10. Aufl., Berlin.

Baron, J./Ritov, I. (1994): Reference Points and Omission Bias, in: Organizational Behavior and Human Decision Processes, Vol. 59, S. 475-498.

Bearden, W. O./Netemeyer, R. G./Teel, J. E. (1989): Measurement of Consumer Susceptibility to Interpersonal Influence, in: Journal of Consumer Research, Vol. 15, S. 473-481.

Bellman, S./Johnson, E. J./Lohse, G. (2001): To opt-in or opt-out: That depends on the Question, in: Communications of the ACM, Vol. 44, S. 25-17.

Boyle, B. F./Dwyer, F. R./Robicheaux, R. A./Simpson, J. T. (1992): Influence Strategies in Marketing Channels: Measures and Use in Different Relationship Structures, in: Journal of Marketing Research, Vol. 29, S. 462-473.

Brown, C. L./Krischna, A. (2004): The Skeptical Shopper: A Metacognitive Account for the Effects of Default Options on Choice, in: Journal of Consumer Research, Vol. 31, S. 529-539.

Brown, S. P. (1990): Use of Closed Influence Tactics by Salespeople: Incidence and Buyers Attributions, in: Journal of Personal Selling \& Sales Management, Vol. 10, S. 17-29.

Campbell, M. C./Kirmani, A. (2000): Consumers' Use of Persuasion Knowledge: The Effects of Accessibility and Cognitive Capacity on Perceptions of an Influence Agent, in: Journal of Consumer Research, Vol. 27, S. 69-83.

Chapman, G. B./Johnson, E. J. (1999): Anchoring, Activation and the Construction of Values, in: Organizational Behavior and Human Decision Processes, Vol. 79, S. 115-153.

Del Veccio, S. K./Zemanek, J. E./McIntyre, R. P./Claxton, R. P. (2003): Buyers Perception of Salesperson Tactical Approaches, in: Journal of Personal Selling \& Sales Management, Vol. 23, S. 39-49.

Desmeules, R. (2002): The Impact of Variety on Consumer Happiness: Marketing and the Tyranny of Freedom, in: Academy of Marketing Science Review, [Online] http://www. amsreview.org/articles/desmeules122002.pdf.

Dhar, R./Wertenbroch, K. (2000): Consumer choice between Hedonic and Utilitarian Goods, in: Journal of Marketing Research, Vol. 37, S. 60-71.

Dubois, B./Laurent, G./Czellar, S. (2004): Segmentation Based on Ambivalent Attitudes, Arbeitspapier, HEC Paris,

Fitzsimons, G. J./Lehmann, D. R. (2004): Reactance to Recommendations: When Unsolicited Advice Yields Contrary Responses, in: Marketing Science, Vol. 23, S. 82-94.

Friestad, M./Wright, P. (1994): The Persuasion Knowledge Model: How People Cope with Persuasion Attempts, in: Journal of Consumer Research, Vol. 21, S. 1-31.

Friestad, M./Wright, P. (1995): Persuasion Knowledge: Lay People's and Researchers' Beliefs about the Psychology of Persuasion, in: Journal of Consumer Research, Vol. 22, S. 62-74.

Friestad, M./Wright, P. (1999): Everyday Persuasion Knowledge, in: Psychology and Marketing, Vol. 16, S. $185-194$.

Gedenk, K. (2002): Verkaufsförderung, München.

Hair, J./Anderson, R./Tatham, R./Black, W. (1998): Multivariate Data Analysis, 5. Aufl., Upper Saddle River.

Heitmann, M./Herrmann, A. (2006): Produktkonfiguration als Präferenzkonstruktion, in: Die Unternehmung, Vol. 60, S. 223-238.

Hsee, C. K./Rottenstreich, Y. (2004): Music, Pandas, and Muggers: On the Affective Psychology of Value, in: Journal of Experimental Psychology: General, Vol. 133, S. 23-30.

Jain, S. P./Posavac, S. S. (2001): Prepurchase Attribute Verifiability, Source Credibility, and Persuasion, in: Journal of Consumer Psychology, Vol. 11, S. 169-180. 
Johnson, E. J./Bellman, S./Lohse, G. L. (2002): Defaults, Framing, and Privacy: Why Opting In - Opting Out, in: Marketing Letters, Vol. 13, S. 5-15.

Johnson, E. J./Goldstein, D. (2003): Do Defaults Save Lifes?, in: Science, Vol. 302, S. 1338-1339.

Johnson, E. J./Herrmann, A./Gächter, S. (2005): The Why, Where and What of Loss Aversion, Arbeitspapier, Columbia University New York.

Johnson, E. J./Hershey, J./Meszaros, J./Kunreuther, H. (1993): Framing, Probability Distortions, and Insurance Decisions, in: Journal of Risk and Uncertainty, Vol. 7, S. 35-51.

Kahneman, D./Knetsch, J. L./Thaler, R. (1990): Experimental Tests of the Endowment Effect and the Coase Theorem, in: Journal of Political Economy, Vol. 98, S. 1325-1348.

Kahneman, D./Tversky, A. (1979): Prospect Theory: An Analysis of Decision Under Risk, in: Econometrica, Vol. 47, 263-291.

Kahneman, D./Tversky, A. (1991): Loss Aversion in Riskless Choice: A Reference-Dependent Model, in: Quarterly Journal of Economics, Vol. S. 1039-1062.

Khan, U./Dhar, R./Wertenbroch, K. (2004): A Behavioral Decision Theoretic Perspective on Hedonic and Utilitarian Choice, Arbeitspapier, Insead, Fontainbleau.

Kivetz, R./Simonson, I. (2002a): Earning the Right to Indulge: Effort as a Determinant of Customer Preferences towards Frequency Program Rewards, in: Journal of Marketing Research, Vol. 39, S. 155-170.

Kivetz, R./Simonson, I. (2002b): Self-Control for the Righteous: Toward a Theory of Precommitment to Indulge, in: Journal of Consumer Research, Vol. 29, S. 199-217.

Levav, J./Heitmann, M./Herrmann, A./Iyengar, S. (2006): The Effect of Variety on sequential Choice, in: Conference of the Institute on Emotions and Decision Making, auf CDrom ohne Seitenangaben.

Levav, J./Heitmann, M./Herrmann, A./Iyengar, S. (2007): The Effect of Attribute Order and Variety on Choice Demotivation: A Field Experiment on German Car Buyers, erscheint in: Advances in Consumer Research.

Madrian, B. C./Shea, D. F. (2001): The Power of Suggestion: Inertia in 401(k) Participation and Savings Behavior, in: Quarterly Journal of Economics, Vol. 66, S. 1149-1187.

McFarland, R. G./Challagalla, G. N./Shervani, T. A. (2006): Influence Tactics for effective adaptive Selling, in: Journal of Marketing, Vol. 70, S. 103-117.

McGuire, W. J. (2000): Standing on the Shoulders of Ancients: Consumer Research, Persuasion, and Figurative Language, in: Journal of Consumer Research, Vol. 27, S. 109-114.

McKenzie, C. R. M. (2004): Framing Effects in Inference Tasks - and why they are normatively defensible, in: Memory and Cognition, Vol. 32, S. 874-885.

McKenzie, C. R. M./Liersch, M. J./Finkelstein, S. R. (2006): Recommendations Implicit in Policy Defaults, in: Psychological Science, Vol. 17, S. 414-420.

McKenzie, C. R. M./Nelson, J. D. (2003): What a Speaker's Choice of Frame Reveals: Reference Points, Frame Selection, and Framing Effects, in: Psychonomic Bulletin and Review, Vol. 10, S. 596-602.

Novemsky, N./Kahneman, D. (2005a): The Boundaries of Loss Aversion, in: Journal of Marketing Research, Vol. 42, S. 119-128.

Novemsky, N./Kahneman, D. (2005b): How Do Intentions Affect Loss Aversion?, in: Journal of Marketing Research, Vol. 42, S. 139-140.

Obermiller, C./Spangenberg, E. R. (1999): Development of a Scale to Measure Consumer Skepticism of Advertising, in: Journal of Consumer Psychology, Vol. 7, S. 159-186.

Park, C. W./Jun, S./MacInnis, D. (2000): Choosing What I Want Versus Rejecting What I do not Want: An Application of Decision Framing to Product Option Choice Decisions, in: Journal of Marketing Research, Vol. 37, S. 187-203.

Payne, J. W./Bettman J. R./Johnson, E. J. (1993): The Adaptive Decision Maker, Cambridge.

Pham, M. T. (1998): Representativeness, Relevance, and the Use of Feelings in Decision Making, in: Journal of Consumer Research, Vol. 25, S. 144-159.

Polak, B./Herrmann, A./Heitmann, M. (2006): Einfluss der Händler auf die Produktspezifikation durch die Kunden, Arbeitspapier, Universität St. Gallen, Forschungsstelle für Business Metrics.

Pornpitakpan, C. (2004): The Persuasiveness of Source Credibility: A Critical Review of five Decades' Evidence, in: Journal of Applied Social Psychology, Vol. 34, S. 243-281.

Roese, N. J. (1997): Counterfactual Thinking, in: Psychological Bulletin, Vol. 121, S. 133-148.

Samuelson, W./Zeckhauser, R. (1988): Status Quo Bias in Decision-Making, in: Journal of Risk and Uncertainty, Vol. 1, S. 7-59.

Sanna, L. J. (1999): Mental Simulations, Affect, and subjective Confidence: Timing is everything, in: Psychological Science, Vol. 10, S. 339-345. 
Schkade, D./Kahneman, D. (1998): Does Living in California make People Happy? A Focusing Illusion in Judgements of Life Satisfaction, in: Psychological Science, Vol. 9, S. 340-346.

Shiv, B./Huber, J. (2000): The Impact of Anticipating Satisfaction on Choice, in: Journal of Consumer resarch, Vol. 27, S. 202-216.

Schweitzer, M. (1994): Disentangling Status Quo and Omission Effects: An Experimental Analysis, in: Organizational Behavior and Human Decision Processes, Vol. 58, 457-476.

Wallsten, T. S./Barton, C. (1982): Processing Probabilistic Multidimensional Information for Decisions, in: Journal of Experimental Psychology: Learning, Memory and Cognition, Vol. 8, S. 361-384.

Wright, P. (2002): Marketplace Metacognition and Social Intelligence, in: Journal of Consumer Research, Vol. 28, S. 677-682.

Zeelenberg, M. (1999): Anticipated Regret, Expected Feedback and Behavioral Decision Making, in: Journal of Behavioral Decision Making, Vol. 12, S. 93-106. 


\section{Die Macht des Defaults - Wirkung von Empfehlungen und Vorgaben auf das individuelle Entscheidungsverhalten}

\section{Zusammenfassung}

Bei der Wahl vieler Produkte ist der Kunde mit Empfehlungen und Vorgaben der Hersteller und Händler (Defaults) konfrontiert. Bei der online-Konfiguration eines Erzeugnisses (z. B. Pkw) ist für alle zwingend erforderlichen Merkmale (z. B. Motor) jeweils ein Default (d. h. eine Ausprägung, z. B. 3.2 Liter) vorgegeben. Zudem geben die Anbieter Empfehlungen bezüglich bestimmter Merkmalsausprägungen ab, an denen sich die Nachfrager orientieren. Obgleich der grundsätzliche Einfluss von Defaults auf das Entscheidungsverhalten unbestritten ist, sind wichtige Fragen etwa nach dem Effekt unterschiedlicher Default-Varianten noch nicht beantwortet. Anhand einer empirischen Studie, in deren Mittelpunkt ein Car-Konfigurator steht, kann gezeigt werden, wie verschiedene Defaults das Entscheidungsverhalten der Individuen beeinflussen. Die Befunde erlauben Rückschlüsse über den Prozess der Herausbildung von Produktpräferenzen.

\section{The Power of Defaults - Effect of defaults on Decision Making}

\section{Summary}

Defaults are those options that customers automatically receive unless they expressly choose an alternative. Should this presetting not correspond to customers' needs, then they must deliberately replace it with an alternative. A key factor in this situation is the individual effort that must be exerted to deselect the default in favour of a more satisfactory alternative product and/or feature. In this paper it is clarified whether the intensity of the default effect changes over varying default steps. In addition, decision-making behavior involves various communicators (e.g., manufacturer, retailer, other customers) that have varying effects on consumer choice of a product, product feature, or feature option (in terms of price and quality), both the default type and the default step are of central significance in explaining such behavior. Finally, customers do not always select the manufacturer and/or retailer defaults, a default may be considered an anchor point that leads consumers to choose a product or product feature options in its proximity. Thus, this default effect is of central significance to the development of a product line and feature options. 